\title{
Data-Based Virtual Unmodeled Dynamics Driven Multivariable Nonlinear Adaptive Switching Control
}

\author{
Tianyou Chai, Fellow, IEEE, Yajun Zhang, Hong Wang, Senior Member, IEEE, Chun-Yi Su, \\ and Jing Sun, Fellow, IEEE
}

\begin{abstract}
For a complex industrial system, its multivariable and nonlinear nature generally make it very difficult, if not impossible, to obtain an accurate model, especially when the model structure is unknown. The control of this class of complex systems is difficult to handle by the traditional controller designs around their operating points. This paper, however, explores the concepts of controller-driven model and virtual unmodeled dynamics to propose a new design framework. The design consists of two controllers with distinct functions. First, using input and output data, a self-tuning controller is constructed based on a linear controller-driven model. Then the output signals of the controller-driven model are compared with the true outputs of the system to produce so-called virtual unmodeled dynamics. Based on the compensator of the virtual unmodeled dynamics, the second controller based on a nonlinear controller-driven model is proposed. Those two controllers are integrated by an adaptive switching control algorithm to take advantage of their complementary features: one offers stabilization function and another provides improved performance. The conditions on the stability and convergence of the closed-loop system are analyzed. Both simulation and experimental tests on a heavily coupled nonlinear twin-tank system are carried out to confirm the effectiveness of the proposed method.
\end{abstract}

Index Terms-Adaptive control, controller-driven model, multivariable and nonlinear systems, switching control, virtual unmodeled dynamics.

\section{INTRODUCTION}

D UE to the complexity and high cost involved in modeling of industrial plants, it is generally difficult to obtain

Manuscript received February 2, 2011; revised June 20, 2011; accepted August 25, 2011. Date of publication November 16, 2011; date of current version December 13, 2011. This work was supported in part by the National Natural Science Foundation of China under Grant 61020106003 and Grant 61074071, Major International (Regional) Joint Research Project: Plant-Wide Optimal Control for Complex Industrial Processes Using a Hybrid DataDriven and Model-Based Approach, the Funds for Creative Research Groups of China under Grant 60821063, Chinese National Fundamental Research Program 2009CB320601, and the 111 Project B08015.

T. Chai and Y. Zhang are with the Key Laboratory of Synthetic Automation for Process Industries, Northeastern University, Shenyang 110819, China (e-mail: tychai@mail.neu.edu.cn (Corresponding Author); zhangyajun79@gmail.com).

H. Wang is with the Control System Center, University of Manchester, Manchester M601QD, U.K. He is also with the Key Laboratory of Synthetic Automation for Process Industries, Northeastern University, Shenyang 110819, China (e-mail: hong.wang@manchester.ac.ak).

C.-Y. Su is with the Department of Mechanical Engineering, Concordia University, Montreal, QC H3G 1M8, Canada (e-mail: cysu@alcor.concordia.ca).

J. Sun is with the Naval Architecture and Marine Engineering Department, University of Michigan, Ann Arbor, MI 48109 USA (e-mail: jingsun@umich.edu).

Color versions of one or more of the figures in this paper are available online at http://ieeexplore.ieee.org.

Digital Object Identifier 10.1109/TNN.2011.2167685 accurate control-oriented mathematical models. At present, most controller designs for complex industrial plants are carried out with a crude model to start with, and use adaptive control and data-driven control to mitigate model uncertainties and modeling errors.

For linear multivariable systems with unknown parameters, a multivariable adaptive decoupling control algorithm has been proposed in [1]-[4] by combining normal decoupling control with self-tuning control. For a class of nonlinear multivariable systems with unknown parameters, an adaptive decoupling control that combines a generalized predictive control with neural network compensation has been developed in [5], where global convergence analysis was not discussed. Using the method in [6], a stable and convergent multivariable adaptive control method was established in [7] for a class of unknown multivariable nonlinear system with stable zero dynamics, where the system was expressed by a combination of unknown linear subsystem and high order nonlinear terms. For a class of unknown multivariable nonlinear systems with unstable zero dynamics, a neural network and multiple-model based dynamic decoupling control algorithm was proposed in [8], where it is shown that the resulting control algorithm is stable and convergent when the $k$-difference for the high order nonlinear term is globally bounded. Furthermore, for a class of industrial processes where the system dynamics are linked with varying production boundary conditions, multiple-model and neural network-based intelligent decoupling control algorithm has been established in [9]. The proposed method in [9] has also been applied to a ball mill coal-pulverizing system of a power plant in China to obtain desired control performance.

However, the methods in [5]-[9] used back-propagation (BP) neural network to approximate the unmodeled dynamics. Since the structure of the BP network is obtained using either experience or trial and error approach [10], the convergence speed of the neural network is slow and it can easily be trapped at a local minimum. This leads to low accuracy of the estimation for unmodeled dynamics and thus affects the control performance.

Besides the adaptive control results mentioned earlier, another important method is data-based controller design, that uses the system input and output data to directly design the controller without requiring a mathematical model of the underlying physical plant. Recently proposed data-driven controller designs include controller falsification [11], [12], simultaneous perturbation stochastic approximation controlbased control design [13], iterative feedback tuning technique 
[14], virtual reference feedback tuning method [15], and datadriven simulation and control [16].

A number of results of data-driven controller design can also be found in literature for multiple-input multiple-output (MIMO) systems. For example, an ellipsoidal unfalsified control method is proposed in [17], where a number of candidate controllers that contain proper unfalsified controllers were first obtained. A performance index was then defined and a desired unfalsified control was selected using the virtual reference output of the pseudo inverse of these controllers. The key issue of this method is the selection of the initial candidate controllers and their pseudo inverses. In [18], a data-driven controller design using correlation analysis has been extended to MIMO cases, where decoupling effect has been realized. However, this method requires that the plant to be control is multivariable time-invariant.

The aforementioned two control design methods (namely the adaptive control and data-driven control), while they differ in many aspects, both assume that the structure of the plant is known a priori while the plant parameters can be unknown. Although the knowledge of the plant structure have not been assumed explicitly in some existing data-driven controller design methods, the knowledge is implicitly assumed in order to select the controller structure. Without this knowledge, it is difficult to obtain a proper control signal to facilitate the tuning of the control parameters, let alone to guarantee good performance and system stability.

Most industrial systems are difficult to model, as they involve multivariable dynamics with complicated nonlinearities, strong couplings, and unknown structure. Moreover, they are subjected to unpredictable disturbances that can drive their dynamical characteristics well-beyond the scope for which the above two types of controllers can cope with. Therefore, it is generally difficult to perform adaptive and data-driven controller design for such plants. It is therefore of significant importance to develop novel control structure and design method using both the knowledge of the plant and controller together with the input and output data of the system. The goal of this paper is to present such a novel design and demonstrate its effectiveness.

The key contributions of the work presented in this paper are briefly stated as follows. This paper mainly focuses on a class of complex multiple-variable industrial systems with unknown structure, for which the mathematical models are very difficult, if not impossible, to establish. To control such systems in industrial setting, a linear model is generally adopted around the operating points and a corresponding linear controller is used. However, owing to the complexities of the system, such a linear controller may not always work. In this case, the unmodeled dynamics have to be taken into consideration. To obtain the unmodeled dynamics, a new controller-driven model is defined first in this paper. Then, the output signals of the controller-driven model are compared with the true outputs of the system to get so-called virtual unmodeled dynamics. An adaptive-network-based fuzzy inference system (ANFIS) is used to estimate and compensate the unmodeled dynamics. Based on the performance indices (11) and (21), a switching function (47) is introduced to switch between the

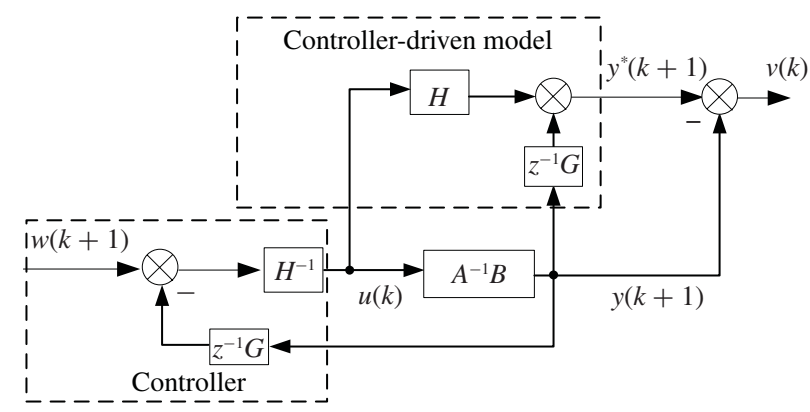

Fig. 1. Structure of the controller and the controller-driven model.

linear controller and the controller with compensator of the unmodeled dynamics to make sure the industrial systems work properly. Furthermore, stability and convergence analyses for this control scheme have been conducted and the corresponding conditions are established. Both simulations and tests on a heavily coupled twin-tank level control system are carried out to verify the effectiveness of the proposed algorithm.

This paper is organized as follows. First, a controllerdriven model and virtual unmodeled dynamics are defined in Section II for general systems. This is then followed by Section III, where the controller-driven model and virtual unmodeled dynamics are proposed for multivariable nonlinear plant whose model are difficult to establish. In Section IV, the development of a linear controller-driven model with self-tuning parameters, an ANFIS-based estimator for virtual unmodeled dynamics and a nonlinear controller-driven model with self-tuning parameters are described. Section V presents the design of the adaptive switching control method. The stability and convergence conditions are derived in Section VI. Finally both simulation and test results on a twin-tank level control system are given in Section VII.

\section{CONCEPTS ON CONTROLler-DRIVEn MODEL AND VIRTUAL UNMODELED DYNAMICS}

To clearly present the concept on controller-driven model and virtual unmodeled dynamics, we consider the following linear time-invariant system:

$$
A\left(z^{-1}\right) y(k+\tau)=A\left(z^{-1}\right) y(k+1)=B\left(z^{-1}\right) u(k)
$$

where $y(k)$ and $u(k)$ are the system output and input at sample time $k$, the time delay is $\tau=1$. Also, $A\left(z^{-1}\right)$ and $B\left(z^{-1}\right)$ are polynomials in terms of the unit time delay operator $z^{-1}$ with the following expressions:

$$
\begin{aligned}
& A\left(z^{-1}\right)=1+a_{1} z^{-1}+\cdots+a_{n_{a}} z^{-n_{a}} \\
& B\left(z^{-1}\right)=b_{0}+b_{1} z^{-1}+\cdots+b_{n_{b}} z^{-n_{b}}
\end{aligned}
$$

where $n_{a}$ and $n_{b}$ are the structure order of the system and $b_{0}$ is a non-zero coefficient. No matter what method the control design is to be used, the control system can be represented as shown in Fig. 1, where the equation of the controller is given by

$$
H\left(z^{-1}\right) u(k)+G\left(z^{-1}\right) y(k)=w(k+1)
$$

where $w(k+1)$ is the desired closed-loop output, $H\left(z^{-1}\right)$ and $G\left(z^{-1}\right)$ are polynomials in terms of $z^{-1}$. 
To design $H\left(z^{-1}\right)$ and $G\left(z^{-1}\right)$, one can apply the controller (2) to (1). This leads to the following closed-loop system equation:

$$
\begin{aligned}
& {\left[H\left(z^{-1}\right) A\left(z^{-1}\right)+z^{-1} B\left(z^{-1}\right) G\left(z^{-1}\right)\right] y(k+1)} \\
& \quad=B\left(z^{-1}\right) w(k+1) .
\end{aligned}
$$

In this context, the purpose of selecting $H\left(z^{-1}\right)$ and $G\left(z^{-1}\right)$ is to guarantee the closed-loop stability and to make $y(k+1)$ to follow $w(k+1)$ as close as possible. This means that

$$
\lim _{k \rightarrow \infty} e(k+1)=\lim _{k \rightarrow \infty}[y(k+1)-w(k+1)]=0 .
$$

From the controller equation in (2), it can be seen that the controller consists of two parts. The left-hand side of the controller equation consists of the system input and output data sequences $\left\{u(k), \ldots, u\left(k-n_{h}\right), y(k), \ldots, y\left(k-n_{g}\right)\right\}$ and the polynomial operators $\left\{H\left(z^{-1}\right), G\left(z^{-1}\right)\right\}$ that represent both the controller structure and the parameters. Such a structure and parameters are determined by the designers of the controller. Therefore in this paper, the left-hand side of (2) is defined as the controller-driven model as shown in Fig. 1. Such a controller-driven model can be expressed as

$$
y^{*}(k+1)=H\left(z^{-1}\right) u(k)+G\left(z^{-1}\right) y(k)
$$

with $y^{*}(k+1)$ being the output of the controller-driven model. It can be seen that once $\left\{H\left(z^{-1}\right), G\left(z^{-1}\right)\right\}$ are obtained, $y^{*}(k+1)$ can be calculated using the system input and output data from (5).

Since the right-hand side of (2) is the pre-specified desired output, this means that any controller can be represented by two parts, namely the controller-driven model and the desired output. This indicates that when the system is accurately described by (1), we should have $y^{*}(k+1)=w(k+1)$.

From the above analysis it can be concluded that the purpose of the selection for $H\left(z^{-1}\right)$ and $G\left(z^{-1}\right)$ is to ensure that when the controller is applied to (1), the closed-loop system is stable and $y(k+1)$ is made as close as possible to $y^{*}(k+1)$.

However, if there are a mismatches between the dynamics of the system to be controlled and the structure and parameters of the controller, there will be a tracking error between the output of the resulting closed-loop system and the desired output of the controller-driven model. This tracking error is expressed as follows:

$$
v(k)=y(k+1)-y^{*}(k+1)
$$

where $v(k)$ is in fact the dynamical tracking error between the closed-loop system output and the controller-driven model output. This term is defined as virtual unmodeled dynamics. Using this concept, a compensator for the virtual unmodeled dynamics can be designed so as to improve the tracking error between the closed-loop output and the desired output caused by the improper selection of the structure and parameters of the controller. Along with this solution route and based upon the fact that complex industrial systems often work near an operating point, a controller-driven model will be first designed using the low order linear model of the system. Then the tracking error between the closed-loop output and the controller-driven model output will be used to obtain a compensator for the virtual unmodeled dynamics. This will form a nonlinear controller with a compensator. A switching mechanism will be established so as to realize a proper switching between linear and nonlinear controllers so that the output of the closed-loop system can follow the desired output as close as possible. Moreover, since the structure and the parameters of the system are unknown, an adaptive switching controller will be formulated using the input and output data together with the tracking error between the output of the closed-loop system and that of the controller-driven model.

\section{Multivariable Controller-Driven Model and ViRTUAL UnMODELED DYNAMICS}

Complex industrial plants that cannot be described by accurate mathematical models can be expressed as the following multivariable nonlinear form:

$$
\begin{aligned}
\boldsymbol{y}(k+1)= & \boldsymbol{f}\left[\boldsymbol{y}(k), \ldots, \boldsymbol{y}\left(k-n_{s}+1\right), \boldsymbol{u}(k),\right. \\
& \left.\boldsymbol{u}(k-1), \ldots, \boldsymbol{u}\left(k-m_{s}\right)\right]+\boldsymbol{d}(k)
\end{aligned}
$$

where $\boldsymbol{u}(k)=\left[u_{1}(k), \ldots, u_{n}(k)\right]^{\mathrm{T}}, \boldsymbol{y}(k)=\left[y_{1}(k), \ldots\right.$, $\left.y_{n}(k)\right]^{\mathrm{T}}$ are the $n$-dimensional system input and output vectors at sample time $k$, respectively. It is assumed that the structure orders $\left(n_{s}, m_{s}\right)$ are unknown and $f(\cdot)$ is an $n$-dimensional unknown vector function and limited to a class of systems, which can be expressed as a linear system and an unmodeled dynamics near the operating points. $\boldsymbol{d}(k)$ is an $n$-dimensional bounded unknown disturbance. It is assumed that the complex industrial systems described by (7) are controllable.

Since the plant of the form (7) often works near an operating point, the following low order linear model can be used for controller design around the corresponding operating point:

$$
\begin{aligned}
\boldsymbol{y}^{*}(k+1)= & -\boldsymbol{A}_{1} \boldsymbol{y}(k)-\cdots-\boldsymbol{A}_{n_{a}} \boldsymbol{y}\left(k-n_{a}+1\right) \\
& +\boldsymbol{B}_{0} \boldsymbol{u}(k)+\cdots+\boldsymbol{B}_{n_{b}} \boldsymbol{u}\left(k-n_{b}\right)
\end{aligned}
$$

where $\boldsymbol{y}^{*}(k+1)=\left[\boldsymbol{y}_{1}^{*}(k+1), \boldsymbol{y}_{2}^{*}(k+1), \ldots, \boldsymbol{y}_{n}^{*}(k+1)\right]^{T}$ are the $n$-dimensional output vector of this model. $\boldsymbol{A}\left(z^{-1}\right)$ and $\boldsymbol{B}\left(z^{-1}\right)$ are polynomial matrices in terms of $z^{-1}$ with structures orders being given by $n_{a}$ and $n_{b}$ which are prespecified by designers. In this context, $\boldsymbol{A}\left(z^{-1}\right)$ and $\boldsymbol{B}\left(z^{-1}\right)$ are denoted as

$$
\begin{array}{r}
\boldsymbol{A}\left(z^{-1}\right)=\boldsymbol{I}+\boldsymbol{A}_{1} z^{-1}+\cdots+\boldsymbol{A}_{n_{a}} z^{-n_{a}} \\
\boldsymbol{B}\left(z^{-1}\right)=\boldsymbol{B}_{0}+\boldsymbol{B}_{1} z^{-1}+\cdots+\boldsymbol{B}_{n_{b}} z^{-n_{b}}
\end{array}
$$

where $\boldsymbol{B}_{0}$ is non-singular.

For a selected linear model (8), the virtual unmodeled dynamics between the output of the model for controller design and that of the plant is then defined as

$$
\boldsymbol{v}(k)=\boldsymbol{f}(\cdot)+\boldsymbol{d}(k)-\boldsymbol{y}^{*}(k+1)
$$

where $\boldsymbol{v}(k)=\left[v_{1}(k), \ldots, v_{n}(k)\right]^{\mathrm{T}}$ is an unknown $n$ dimensional nonlinear vector that includes various disturbances and unmodeled dynamics.

From (7)-(9), it can be obtained that

$$
\begin{aligned}
\boldsymbol{y}(k+1)= & \boldsymbol{y}^{*}(k+1)+\boldsymbol{v}(k) \\
= & -\boldsymbol{A}_{1} \boldsymbol{y}(k)-\cdots-\boldsymbol{A}_{n_{a}} \boldsymbol{y}\left(k-n_{a}+1\right)+\boldsymbol{B}_{0} \boldsymbol{u}(k) \\
& +\cdots+\boldsymbol{B}_{n_{b}} \boldsymbol{u}\left(k-n_{b}\right)+\boldsymbol{v}(k) .
\end{aligned}
$$


Therefore we have

$$
\boldsymbol{A}\left(z^{-1}\right) \boldsymbol{y}(k+1)=\boldsymbol{B}\left(z^{-1}\right) \boldsymbol{u}(k)+\boldsymbol{v}(k) .
$$

To design the required controller, the following performance index is used:

$$
J=\left\|\boldsymbol{y}(k+1)-\left[\boldsymbol{w}(k+1)-\boldsymbol{Q}\left(z^{-1}\right) \boldsymbol{u}(k)\right]\right\|^{2}
$$

where $\boldsymbol{Q}\left(z^{-1}\right)$ are an $n \times n$ weighting polynomial matrix in terms of $z^{-1}$ and is selected as follows:

$$
\begin{aligned}
\boldsymbol{Q}\left(z^{-1}\right) & =\boldsymbol{Q}^{*} \cdot\left(1-z^{-1}\right) \\
\boldsymbol{Q}^{*} & =\left[\begin{array}{ccccc}
q_{11} & q_{12} & \cdots & q_{1(n-1)} & q_{1 n} \\
q_{21} & q_{22} & \cdots & q_{2(n-1)} & q_{2 n} \\
\vdots & \vdots & \ddots & \vdots & \vdots \\
q_{(n-1) 1} & q_{(n-1) 2} & \cdots & q_{(n-1)(n-1)} & q_{(n-1) n} \\
q_{n 1} & q_{n 2} & \cdots & q_{n(n-1)} & q_{n n}
\end{array}\right]
\end{aligned}
$$

where $q_{i l}(i, l=1,2, \ldots, n)$ are constants chosen off-line via a trial and error method so that they satisfy the following inequality:

$$
\operatorname{det}\left[\boldsymbol{B}\left(z^{-1}\right)+\boldsymbol{A}\left(z^{-1}\right) \boldsymbol{Q}^{*}\left(1-z^{-1}\right)\right] \neq 0, \quad|z|>1
$$

At this stage, a generalized desired output vector $\phi^{*}(k+1)$ can be defined to read

$$
\phi^{*}(k+1)=\boldsymbol{w}(k+1)-\boldsymbol{Q}\left(z^{-1}\right) \boldsymbol{u}(k) .
$$

Moreover, one can select $\boldsymbol{G}\left(z^{-1}\right)$ so that the following Diophantine equality holds:

$$
\boldsymbol{I}=\boldsymbol{A}\left(z^{-1}\right)+z^{-1} \boldsymbol{G}\left(z^{-1}\right)
$$

where the order of $\boldsymbol{G}\left(z^{-1}\right)$ is $n_{g}=n_{a}-1$.

From (10) and (15), it can be obtained that

$$
\boldsymbol{y}(k+1)=\boldsymbol{G}\left(z^{-1}\right) \boldsymbol{y}(k)+\boldsymbol{H}\left(z^{-1}\right) \boldsymbol{u}(k)+\boldsymbol{v}(k)
$$

where $\boldsymbol{H}\left(z^{-1}\right)$ is an $n_{b}$ th order polynomial matrix in terms of $z^{-1}$ [i.e., $\left.\boldsymbol{H}\left(z^{-1}\right)=\boldsymbol{B}\left(z^{-1}\right)\right]$.

It can be seen that the optimal prediction of $\boldsymbol{y}(k+1)$ should be expressed as

$$
\phi^{*}(k+1 \mid k)=\boldsymbol{G}\left(z^{-1}\right) \boldsymbol{y}(k)+\boldsymbol{H}\left(z^{-1}\right) \boldsymbol{u}(k) .
$$

Using (14) and (17), the linear controller equation that optimizes (11) can be formulated to read

$$
\boldsymbol{G}\left(z^{-1}\right) \boldsymbol{y}(k)+\boldsymbol{H}\left(z^{-1}\right) \boldsymbol{u}(k)=\boldsymbol{w}(k+1)-\boldsymbol{Q}^{*}\left(1-z^{-1}\right) \boldsymbol{u}(k) .
$$

Meanwhile, the linear multivariable controller-driven model, in the form of (5), should be given by

$$
\boldsymbol{y}^{*}(k+1)=\boldsymbol{G}\left(z^{-1}\right) \boldsymbol{y}(k)+\boldsymbol{H}\left(z^{-1}\right) \boldsymbol{u}(k) .
$$

Therefore, from (16) and (19), the virtual unmodeled dynamics can be obtained as follows:

$$
\begin{aligned}
\boldsymbol{y} & (k+1)-\boldsymbol{y}^{*}(k+1) \\
& =\boldsymbol{y}(k+1)-\left[\boldsymbol{G}\left(z^{-1}\right) \boldsymbol{y}(k)+\boldsymbol{H}\left(z^{-1}\right) \boldsymbol{u}(k)\right] \\
& =\boldsymbol{v}(k) .
\end{aligned}
$$

To design a compensator for $\boldsymbol{v}(k)$ in the form of $\boldsymbol{K}\left(z^{-1}\right) \boldsymbol{v}(k)$, the following performance index is selected [9]:

$$
J=\left\|\boldsymbol{y}(k+1)-\left[\boldsymbol{w}(k+1)-\boldsymbol{Q}\left(z^{-1}\right) \boldsymbol{u}(k)-\boldsymbol{K}\left(z^{-1}\right) \boldsymbol{v}(k)\right]\right\|^{2}
$$

where

$$
\begin{aligned}
\boldsymbol{K}\left(z^{-1}\right) & =\boldsymbol{K}^{*} \cdot\left(1-z^{-1}\right) \\
\boldsymbol{K}^{*} & =\operatorname{diag}\left\{k_{1}, k_{2}, \ldots, k_{n}\right\}
\end{aligned}
$$

with $k_{l}(l=1,2, \ldots, n)$ being constants to be specified.

In this case, the generalized output becomes

$\phi^{*}(k+1)=\boldsymbol{w}(k+1)-Q^{*}\left(1-z^{-1}\right) \boldsymbol{u}(k)-\boldsymbol{K}^{*}\left(1-z^{-1}\right) \boldsymbol{v}(k)$.

In a similar way, the nonlinear multivariable controller equation that optimizes (21) can be formulated to give

$$
\begin{aligned}
& \boldsymbol{G}\left(z^{-1}\right) \boldsymbol{y}(k)+\boldsymbol{H}\left(z^{-1}\right) \boldsymbol{u}(k)+\boldsymbol{v}(k) \\
& \quad=\boldsymbol{w}(k+1)-\left[\boldsymbol{Q}^{*}\left(1-z^{-1}\right) \boldsymbol{u}(k)+\boldsymbol{K}^{*}\left(1-z^{-1}\right) \boldsymbol{v}(k)\right] .
\end{aligned}
$$

Therefore, the multivariable nonlinear controller-driven model is given by

$$
\boldsymbol{y}_{2}^{*}(k+1)=\boldsymbol{G}\left(z^{-1}\right) \boldsymbol{y}(k)+\boldsymbol{H}\left(z^{-1}\right) \boldsymbol{u}(k)+\boldsymbol{v}(k) .
$$

Since the parameters of $\boldsymbol{G}\left(z^{-1}\right)$ and $\boldsymbol{H}\left(z^{-1}\right)$ are unknown, $\boldsymbol{v}(k)$ is also unknown. Therefore, the input and output data should be used to estimate the virtual unmodeled dynamics $\boldsymbol{v}(k)$ and the parameter of the controller-driven model (19) and (25).

\section{Controller-Driven Models With SElF-Tuning PARAMETERS AND THE ESTIMATION OF VIRTUAL UNMODELED DYNAMICS}

\section{A. Linear Controller-Driven Model with Self-Tuning Parame-} ters

It can be seen that (16) can be turned into the parameter estimation equation for the linear controller-driven model in the form of

$\boldsymbol{y}(k+1)=\boldsymbol{G}\left(z^{-1}\right) \boldsymbol{y}(k)+\boldsymbol{H}\left(z^{-1}\right) \boldsymbol{u}(k)+\boldsymbol{v}(k)=\boldsymbol{\Theta}^{\mathrm{T}} \boldsymbol{x}(k)+\boldsymbol{v}(k)$

where

$$
\begin{aligned}
\boldsymbol{G}\left(z^{-1}\right):= & \boldsymbol{G}_{0}+\boldsymbol{G}_{1} z^{-1}+\cdots+\boldsymbol{G}_{n_{a}-1} z^{-n_{a}+1}, \\
\boldsymbol{H}\left(z^{-1}\right)= & \boldsymbol{H}_{0}+\boldsymbol{H}_{1} z^{-1}+\cdots+\boldsymbol{H}_{n_{b}} z^{-n_{b}}, \\
\boldsymbol{\Theta}= & {\left[\boldsymbol{G}_{0}, \ldots, \boldsymbol{G}_{n_{a}-1}, \boldsymbol{H}_{0}, \ldots, \boldsymbol{H}_{n_{b}}\right]^{\mathrm{T}}, } \\
\boldsymbol{x}(k)= & {\left[x_{1}(k), x_{2}(k), \ldots, x_{n \times\left(n_{a}+n_{b}+1\right)}(k)\right]^{\mathrm{T}} } \\
= & {\left[\boldsymbol{y}(k)^{\mathrm{T}}, \ldots, \boldsymbol{y}\left(k-n_{a}+1\right)^{\mathrm{T}},\right.} \\
& \left.\boldsymbol{u}(k)^{\mathrm{T}}, \ldots, \boldsymbol{u}\left(k-n_{b}\right)^{\mathrm{T}}\right]^{\mathrm{T}} .
\end{aligned}
$$

With (26), the following algorithm can be used to estimate $\boldsymbol{\Theta}$ :

$$
\hat{\boldsymbol{\Theta}}_{1}(k)=\hat{\boldsymbol{\Theta}}_{1}(k-1)+\boldsymbol{h}(k) \boldsymbol{e}_{1}(k)^{T}
$$




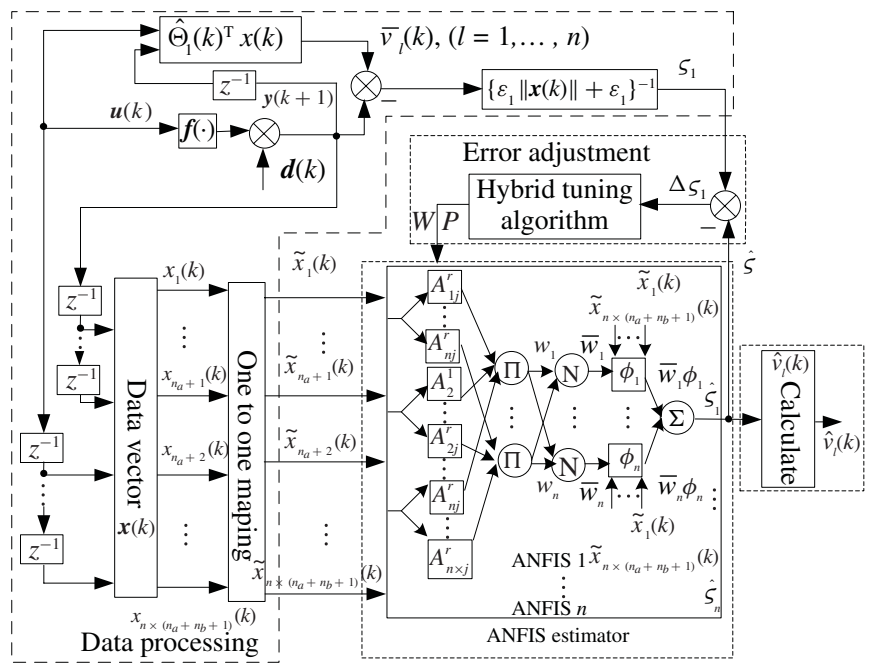

Fig. 2. Structure of the estimation for the virtual unmodeled dynamic $v(k)$

where $\boldsymbol{h}(k)$ can be selected to be in either the least square estimation or parameter projection format, and $\hat{\boldsymbol{\Theta}}_{1}(k)$ is the estimate of $\boldsymbol{\Theta}$. The estimation error $\boldsymbol{e}_{1}(k)$ is defined as

$$
\boldsymbol{e}_{1}(k)=\boldsymbol{y}(k)-\hat{\boldsymbol{y}}(k)
$$

where $\hat{\boldsymbol{y}}(k)=\hat{\boldsymbol{\Theta}}_{1}(k-1)^{T} \boldsymbol{x}(k-1)$.

This means that the linear controller-driven model with selftuning parameters can be expressed by

$$
\hat{\boldsymbol{y}}(k+1)=\hat{\boldsymbol{\Theta}}_{1}(k)^{T} \boldsymbol{x}(k)
$$

where $\hat{\boldsymbol{\Theta}}_{1}(k)$ is given by (28) and (29).

\section{B. Estimation of Virtual Unmodeled Dynamics}

From (28)-(30), the output of the controller-driven model with self-tuning parameters can be obtained. Therefore, the difference between the output of the closed-loop system and that of the controller-driven model can be expressed as

$$
\overline{\boldsymbol{v}}(k)=\boldsymbol{y}(k+1)-\hat{\boldsymbol{\Theta}}_{1}(k)^{T} \boldsymbol{x}(k) .
$$

Using the input and output data together with $\bar{v}(k)$, the virtual unmodeled dynamics can be estimated using an estimator as shown in Fig. 2, which consists of data processing, ANFIS [19] based estimation, the error adjustment, and the estimation for $\boldsymbol{v}(k)$.

1) Data Processing: At first, a normalization of $\overline{\boldsymbol{v}}(k)$ can produce $\varsigma(k)$, its $l$ th $(l=1, \ldots, n)$ component should satisfy $\left|s_{l}(k)\right| \leq 1$. Therefore, the following can be obtained:

$$
\varsigma_{l}(k)=\frac{\bar{v}_{l}(k)}{\varepsilon_{1}\|\boldsymbol{x}(k)\|+\varepsilon_{2}}
$$

where $\|x(\boldsymbol{k})\|=\left\{x^{\mathrm{T}}(\boldsymbol{k}) x(\boldsymbol{k})\right\}^{1 / 2}, 0 \leq \varepsilon_{1}<1$, and $\varepsilon_{2}>0$ are constants.

Moreover, to ensure the boundedness of the input vector $\boldsymbol{x}(k)$ for the ANFIS estimator, the one-to-one mapping $\alpha$ in [20] can be applied to $\boldsymbol{x}(k)$ to obtain $\tilde{\boldsymbol{x}}(k)$, whose $i$ th component is denoted as $\tilde{x}_{i}(k)$ which can be calculated from

$$
\tilde{x}_{i}(k)=\alpha\left[x_{i}\right]=\frac{1}{1+\exp \left[-x_{i}\right]}
$$

where $i=1,2, \ldots, n \times\left(n_{a}+n_{b}+1\right)$. It can be seen that after (33), $\tilde{\boldsymbol{x}}(k)$ is a uniformly bounded vector that are defined in a compact set.

2) ANFIS-Based Estimator: In order to construct the ANFIS-based estimator, $\tilde{\boldsymbol{x}}(k)$ is first fuzzified, where the $i$ th component $\tilde{x}_{i}(k)$ is divided into $m_{1}$ fuzzy set. The $j$ th fuzzy set of $\tilde{x}_{i}(k)$ is represented by $A_{i j}\left(j=1,2, \ldots, m_{1}\right)$ with the following membership function:

$$
\rho_{i j}\left(\tilde{x}_{i}(k)\right)=\exp \left[-\frac{\left(\tilde{x}_{i}(k)-c_{i j}\right)^{2}}{2\left(\sigma_{i j}\right)^{2}}\right]
$$

where $c_{i j}$ and $\sigma_{i j}$ are the centers and width of the membership function, respectively, and are tuned based on the estimation error.

Using the above fuzzification, a number of $m=m_{1} \times n \times$ $\left(n_{a}+n_{b}+1\right)$ fuzzy rules can be obtained, where any ruler $(r=$ $1, \ldots, m)$ denoted by $R^{r}$, can be expressed as [21] and [22].

$R^{r}:$ If $\tilde{x}_{1}(k)$ is $A_{1 j}^{r}, \tilde{x}_{2}(k)$ is $A_{2 j}^{r}, \ldots, \tilde{x}_{n \times\left(n_{a}+n_{b}+1\right)}(k)$ is $A_{n \times 1}^{r}$

$n \times\left(n_{a}+n_{b}+1\right), j$.

Then

$$
\phi_{r}(k)=\sum_{i=0}^{n \times\left(n_{a}+n_{b}+1\right)} p_{i j}^{r} \tilde{x}_{i}(k)
$$

where the $j$ th fuzzy set of the $\tilde{x}_{i}(k)$ division for the $r$ th rule is denoted as $A_{i j}^{r}$, whose membership function is denoted by $\rho_{i j}^{r}\left(\tilde{x}_{i}(k)\right)$. In the above equation $p_{i j}^{r}$ are the linking weights. When $i=0, p_{i j}^{r}=p_{0 j}^{r}, \tilde{x}_{0}(k)=1$, and $p_{i j}^{r}$ is tuned using the estimation error. Therefore, the estimation for $\varsigma_{l}(k)$ is given by

$$
\hat{\varsigma}_{l}(k)=\sum_{r=1}^{m} \bar{w}_{r}(k) \phi_{r}(k), \quad l=1,2, \ldots, n
$$

where $\bar{w}_{r}(k)=\left(w_{r}(k) / \sum_{r=1}^{m} w_{r}(k)\right), \quad w_{r}(k)=$ $\prod_{i=1}^{n \times\left(n_{a}+n_{b}+1\right)} \mu_{i j}^{r}\left(\tilde{x}_{i}(k)\right) \quad$ with parameters $c_{i j}^{r}$ and $\sigma_{i j}^{r}$ being tuned again using the estimation error.

3) Tuning of the Estimation Error: From the above formulation, it can be seen that the estimation error is given by

$$
\Delta \varsigma_{l}(k)=\varsigma_{l}(k)-\hat{\varsigma}_{l}(k) \quad(l=1,2, \ldots, n) .
$$

Therefore, the tuning of $p_{i j}^{r}$ can be obtained to read

$$
\Delta \varsigma_{l}(k)=\varsigma_{l}(k)-\sum_{r=1}^{m}\left[\bar{w}_{r} \sum_{i=0}^{n \times\left(n_{a}+n_{b}+1\right)} p_{i j}^{r} \tilde{x}_{i}(k)\right] .
$$

For a fixed $\bar{w}_{r}$, the recursive least square estimation in [19] can be used to evaluate the tuned value $p_{i j}^{r}(k)$ for $p_{i j}^{r}$. Using the tuned value of $p_{i j}^{r}(k), c_{i j}^{r}$, and $\sigma_{i j}^{r}$ can also be adjusted online. For this purpose, the following performance index is defined:

$$
\begin{aligned}
E & =\frac{1}{2} \Delta \varsigma_{l}^{2} \\
& =\frac{1}{2}\left|\varsigma_{l}(k)-\sum_{r=1}^{m} \frac{w_{r}(k) \sum_{i=0}^{n \times\left(n_{a}+n_{b}+1\right)} p_{i j}^{r}(k) \tilde{x}_{i}(k)}{\sum_{i=1}^{m \times\left(n_{a}+n_{b}+1\right)} \exp \left[-\frac{\left(\tilde{x}_{i}(k)-c_{i j}^{r}\right)^{2}}{2\left(\sigma_{i j}^{r}\right)^{2}}\right]}\right|^{2}
\end{aligned}
$$

where " $|*|$ " denotes the absolute value of the elements of " $*$." 
Using the gradient descent rules in [19] to minimize (36), the adjusted value for $c_{i j}^{r}$ can be obtained to give $c_{i j}^{r}(k)$.

Using both $p_{i j}^{r}(k)$ and $c_{i j}^{r}(k)$ and the following equation:

$$
E=\frac{1}{2}\left|\varsigma_{l}(k)-\sum_{r=1}^{m} \frac{w_{r}(k) \sum_{i=0}^{n \times\left(n_{a}+n_{b}+1\right)} p_{i j}^{r}(k) \tilde{x}_{i}(k)}{\sum_{i=1}^{m \times\left(n_{a}+n_{b}+1\right)} \exp \left[-\frac{\left(\tilde{x}_{i}(k)-c_{i j}^{r}(k)\right)^{2}}{2\left(\sigma_{i j}^{r}\right)^{2}}\right]}\right|^{2}
$$

the adjusted value for $\sigma_{i j}^{r}$ can be readily obtained by minimizing the above performance index using again the gradient rule [19].

4) Estimation of Virtual Unmodeled Dynamics: From (32) it can be obtained that

$$
\bar{v}_{l}(k)=\varsigma_{l}(k)\left(\varepsilon_{1}\|\boldsymbol{x}(k)\|+\varepsilon_{2}\right) .
$$

As such, the following equality can be formulated from (33):

$$
x_{i}(k)=\alpha^{-1}\left(\tilde{x}_{i}(k)\right)=-\ln \left(\frac{1}{\tilde{x}_{i}(k)}-1\right) .
$$

Therefore $\hat{v}_{l}(k)(l=1,2, \ldots, n)$ can be calculated from

$$
\hat{v}_{l}(k)=\hat{\varsigma}_{l}(k)\left[\varepsilon_{1}\left\|\alpha^{-1}[\tilde{\boldsymbol{x}}(k)]\right\|+\varepsilon_{2}\right]
$$

where

$$
\begin{aligned}
\alpha^{-1}[\tilde{\boldsymbol{x}}(k)]= & {\left[\alpha ^ { - 1 } \left(\tilde{x}_{1}(k), \ldots, \alpha^{-1}\left(\tilde{x}_{i}(k), \ldots, \alpha^{-1}\right.\right.\right.} \\
& \left.\left(\tilde{x}_{n \times\left(n_{a}+n_{b}+1\right)}(k)\right)\right] .
\end{aligned}
$$

Remark 1: When using the proposed method to estimate the virtual unmodeled dynamics, for any $x_{i}(k) \in(-\infty,+\infty)$, (33) indicates that $\tilde{x}_{i}(k) \in(0,1) \subset[0,1]$. This means that $\tilde{x}_{i}(k)$ belongs to a compact set. Therefore the input to the ANFIS also belongs to a compact set. Using the well-known universal approximation properties of the fuzzy systems [23], it can be concluded that for any continuous function $\overline{\boldsymbol{v}}(k)$ [i.e., $\overline{\boldsymbol{v}}(k)$ stands for $\overline{\boldsymbol{v}}(x(k))]$ and any $\xi>0$, there exists a desired fuzzy system so that

$$
\| \overline{\boldsymbol{v}}(k)-\hat{\boldsymbol{v}}(k)] \| \leq \xi
$$

where $\xi$ is a pre-specified and arbitrarily small positive number.

Remark 2: In the proposed method, the major computational complexity is the implementation of the ANFIS that is used to estimate the virtual-unmodeled dynamic of the system. In fact, some functions in the ANFIS, such as genfis1, genfis2, and other standard modules, can be trained in advance using MATLAB Fuzzy Logic Toolbox, making the ANFIS implementation in real time straightforward. In this case, the computational complexity for implementation of the ANFIS is alleviated.

\section{Nonlinear Controller-Driven Model with Self-Tuning Para- meters}

By subtracting both sides of (26) with $\hat{\boldsymbol{v}}(k)$, it can be obtained that

$$
\boldsymbol{y}(k+1)-\hat{\boldsymbol{v}}(k)=\boldsymbol{\Theta}^{\mathrm{T}} \boldsymbol{x}(k)+\Delta \boldsymbol{v}(k)
$$

where $\Delta \boldsymbol{v}(k)=\boldsymbol{v}(k)-\hat{\boldsymbol{v}}(k)$.
Then the following estimation algorithm can be used to estimate $\boldsymbol{\Theta}^{\mathrm{T}}$ :

$$
\hat{\boldsymbol{\Theta}}_{2}(k)=\hat{\boldsymbol{\Theta}}_{2}(k-1)+\boldsymbol{h}(k) \boldsymbol{e}_{2}(k)^{\mathrm{T}}
$$

where term $\boldsymbol{h}(k)$ can be either of the least square or project format. Therefore, $\hat{\boldsymbol{\Theta}}_{2}(k)$ can be seen as the estimated value of $\boldsymbol{\Theta}$ for the nonlinear controller-driven model at sample time $k$ with the following estimation error:

$$
\boldsymbol{e}_{2}(k)=\boldsymbol{y}(k)-\hat{\boldsymbol{v}}(k-1)-\hat{\boldsymbol{\Theta}}_{2}(k-1)^{T} \boldsymbol{x}(k-1) .
$$

The nonlinear controller-driven model with self-tuning parameters can be expressed as follows:

$$
\hat{\boldsymbol{y}}(k+1)=\hat{\boldsymbol{\Theta}}_{2}(k)^{T} \boldsymbol{x}(k)+\hat{\boldsymbol{v}}(k)
$$

where $\hat{\boldsymbol{\Theta}}_{2}(k)$ is tuned using (40) and (41).

\section{Adaptively Switching Control Algorithm}

\section{A. Controller Equation with Self-Tuning Parameters}

From (18), (19), and (26), it can be seen that the linear controller (18) can be expressed as

$$
\boldsymbol{\Theta}^{\mathrm{T}} \boldsymbol{x}(k)=\boldsymbol{w}(k+1)-\boldsymbol{Q}^{*}\left(1-z^{-1}\right) \boldsymbol{u}(k)
$$

where the left-hand side constitutes the controller-driven model while the right-hand side is the generalized desired output as given in (14).

By using the identification algorithm (28) and (29) to estimate parameter $\boldsymbol{\Theta}$ of the controller-driven model, its output can be obtained to read $\hat{\boldsymbol{\Theta}}_{1}(k)^{T} \boldsymbol{x}(k)$ which can be made to be equal to the generalized desired output in (14) so as to obtain the following linear adaptive controller equation:

$$
\hat{\boldsymbol{\Theta}}_{1}(k)^{T} \boldsymbol{x}(k)=\boldsymbol{w}(k+1)-\boldsymbol{Q}^{*}\left(1-z^{-1}\right) \boldsymbol{u}(k) .
$$

Therefore, the nonlinear controller equation can be formulated from (24) and (26) as follows:

$$
\begin{aligned}
\boldsymbol{\Theta}^{\mathrm{T}} \boldsymbol{x}(k)+\boldsymbol{v}(k)= & \boldsymbol{w}(k+1)-\boldsymbol{Q}^{*}\left(1-z^{-1}\right) \boldsymbol{u}(k) \\
& -\boldsymbol{K}^{*}\left(1-z^{-1}\right) \boldsymbol{v}(k) .
\end{aligned}
$$

It can be seen that the left-hand side of (23) and (25) constitutes the nonlinear controller-driven model while the right-hand side is the generalized desired output.

Since the nonlinear controller-driven model parameter $\hat{\boldsymbol{\Theta}}_{2}(k)$ can be estimated using the identification algorithm in (40) and (41), the estimate of the virtual unmodeled dynamics $\hat{\boldsymbol{v}}(k)$ can be readily obtained using (31)-(37). By making the nonlinear controller-driven model with selftuning parameter (42) equal to the generalized desired output in (23), the following nonlinear adaptive controller can be obtained:

$$
\begin{aligned}
\hat{\boldsymbol{\Theta}}_{2}(k)^{\mathrm{T}} \boldsymbol{x}(k)+\hat{\boldsymbol{v}}(k)= & \boldsymbol{w}(k+1)-\boldsymbol{Q}^{*}\left(1-z^{-1}\right) \boldsymbol{u}(k) \\
& -\boldsymbol{K}^{*}\left(1-z^{-1}\right) \hat{\boldsymbol{v}}(k) .
\end{aligned}
$$




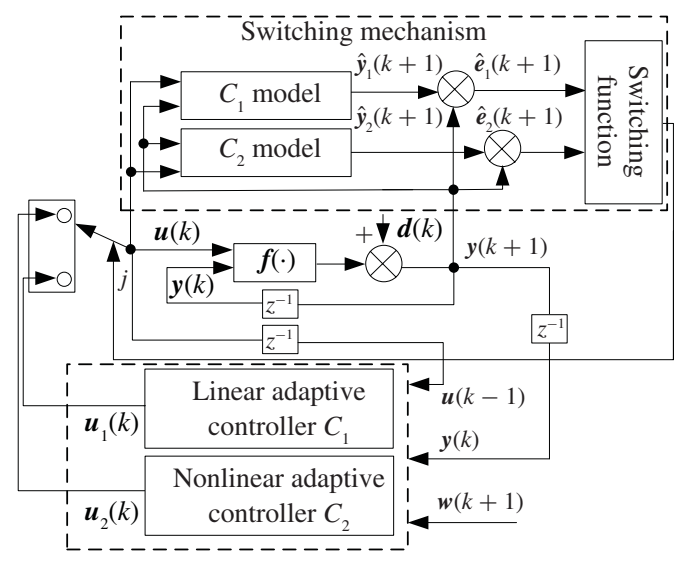

Fig. 3. Structure of adaptive switching control.

\section{B. Adaptive Switching Control}

From (29), (30), (41), and (42), it can be seen that the estimation errors $\boldsymbol{e}_{1}(k)$ and $\boldsymbol{e}_{2}(k)$ of model parameters represent the tracking errors between the output of the closedloop system and that of the linear controller-driven model with self-tuning parameter, and the error between the output of the closed-loop system and that of the nonlinear controllerdriven model, respectively. The smaller these estimation errors are, the better the tracking performance for the closed-loop system. Therefore, $\boldsymbol{e}_{1}(k)$ and $\boldsymbol{e}_{2}(k)$ can be embedded into a switching mechanism, where the active controller (and the corresponding controller-driven-model) can be selected from the one that produces the smallest tracking error between the output of the closed-loop system. In this paper, a switching mechanism is designed as shown in Fig. 3 that consists of a switching function together with $C_{1}$ and $C_{2}$, where $C_{1}$ stands for the linear adaptive controller based on controller-driven model while $C_{2}$ represents the nonlinear adaptive controller based on nonlinear controller-driven model.

The idea here is to use linear adaptive controller $C_{1}$ to ensure the uniform boundedness of the input and output signals in the closed-loop system while use nonlinear adaptive controller $C_{2}$ to improve the system performance whenever we can. In doing so, the switching mechanism performs a proper selection of these two adaptive controllers so that both the uniform boundedness of input and output and the dynamical performance of the closed-loop can be guaranteed at the same time.

The switching function is selected using the method in [7] to evaluate

$$
\begin{aligned}
J_{j}(k)= & \sum_{t=1}^{k} \frac{\mu_{j}(t)\left[\left\|\boldsymbol{e}_{j}(t)\right\|^{2}-M^{2}\right]}{2\left[1+\boldsymbol{x}(t-1)^{\mathrm{T}} \boldsymbol{x}(t-1)\right]}+c\left[1-\mu_{j}(t)\right] \\
& \times\left\|\boldsymbol{e}_{j}(t)\right\|^{2} \\
\mu_{j}(k)= & \left\{\begin{array}{ll}
1, \quad\left\|\boldsymbol{e}_{j}(k)\right\|>M, \\
0, \quad \text { otherwise }
\end{array} \quad j=1,2\right.
\end{aligned}
$$

where $j=1,2$. When $j=1, \boldsymbol{e}_{1}(k)$ represents the estimation error for the linear adaptive controller $C_{1}$ based on linear controller-driven model. When $j=2, \boldsymbol{e}_{2}(k)$ stands for the estimation error of the nonlinear adaptive controller $C_{2}$ based on nonlinear controller-driven model. In the above equation, $c \geq 0, M$ will be defined. $\boldsymbol{e}_{1}(k)$ and $\boldsymbol{e}_{2}(k)$ are calculated from (29) and (41), respectively.

In this paper, for any sample time $k$, the switching mechanism would select the controller that corresponds to the minimum switching function for the system. This is illustrated as follows:

$$
J_{\min }(k)=\operatorname{Min}\left[J_{1}(k), J_{2}(k)\right]
$$

when $J_{\min }(k)=J_{1}(k), \boldsymbol{u}_{1}(k)$ is selected for the system, when $J_{\min }(k)=J_{2}(k), \boldsymbol{u}_{2}(k)$ is applied to the system.

\section{Adaptive Switching Algorithm}

To summarize, the following algorithm is obtained.

Step 1: Use the measured input and output data $\left\{\boldsymbol{y}(k), \ldots, \boldsymbol{y}\left(k-n_{a}+1\right), \boldsymbol{u}(k-1), \ldots, \boldsymbol{u}\left(k-n_{b}\right)\right\}$ and (27) to construct data vector $\boldsymbol{x}(k)$.

Step 2: Use identification algorithm (28) and (29) to obtain the estimation $\hat{\boldsymbol{\Theta}}_{1}(k)$ of the controller-driven model with self-tuning parameters, and then to produce $\boldsymbol{u}_{1}(k)$ using controller (44).

Step 3: Obtain $\hat{\boldsymbol{v}}(k)$ which is the estimation of the virtual unmodeled dynamics $\boldsymbol{v}(k)$ by using the data-driven estimation algorithm (31)-(37).

Step 4: Calculate the estimated value $\hat{\boldsymbol{\Theta}}_{2}(k)$ for the parameters of the self-tuning nonlinear controller-driven model using the identification algorithm in (40) and (41), and then obtain $\boldsymbol{u}_{2}(k)$ from the controller (46).

Step 5: Calculate the estimation errors $\boldsymbol{e}_{1}(k)$ and $\boldsymbol{e}_{2}(k)$ using (29) and (41), respectively, and then use switching functions (47) and (48) to evaluate $J_{1}(k)$ and $J_{2}(k)$ so as to obtain $J_{\min }(k)$.

Step 6: Select the control signal $\boldsymbol{u}(k)$ that corresponds to $J_{\min }(k)$ for the system (1) so as to generate the new system output $\boldsymbol{y}(k+1)$.

Step 7: Let $k=k+1$, and then go back to Step 1 .

\section{Stability and Convergence Analysis}

In this section, the stability and convergence of the closedloop system with the proposed controller will be analyzed.

First, we should clarify that the proposed algorithm is designed to deal with a class of multivariable complex industrial systems described by (7) with unknown model structure orders and parameters. Basically, we assume that such systems work near their operating points and can be expressed by a low-order linear system and unmodeled dynamics.

Second, the stability and convergence analysis presented in this paper are carried out under the following conditions.

Condition 1: The virtual unmodeled dynamics $\boldsymbol{v}(k)$ satisfies the following inequality:

$$
\|\boldsymbol{v}(k)\| \leq \gamma(k), \quad \forall k
$$

where

$$
\gamma(k)=\varepsilon_{1}\|\boldsymbol{x}(k)\|+\varepsilon_{2}
$$

$0 \leq \varepsilon_{1}<1$ and $\varepsilon_{2}>0$ are constants, which are the same as in (32). 
Condition 2: The parameter estimation for the linear controller-driven model uses the following projection algorithm:

$$
\hat{\boldsymbol{\Theta}}_{1}(k)=\operatorname{proj}\left\{\hat{\boldsymbol{\Theta}}_{1}^{\prime}(k)\right\}\left\{\begin{array}{l}
\hat{\boldsymbol{\Theta}}_{1}^{\prime}(k), \operatorname{det}\left[\hat{\mathbf{H}}_{0}(k)\right] \neq 0 \\
{\left[\ldots, \hat{\mathbf{H}}_{0}(k-1), \ldots\right]^{T}, \quad \text { otherwise }}
\end{array}\right.
$$

where $\hat{\boldsymbol{\Theta}}_{1}^{\prime}(k)$ is calculated form (28) and (29), therein $\boldsymbol{h}(k)$ is calculated as follows:

$$
\begin{aligned}
\boldsymbol{h}(k) & =\frac{\mu_{1}(k) \boldsymbol{x}(k-1)}{1+\boldsymbol{x}(k-1)^{T} \boldsymbol{x}(k-1)} \\
\mu_{1}(k) & = \begin{cases}1, & \left\|\boldsymbol{e}_{1}(k)\right\|>2 \gamma(k-1) \\
0, & \text { otherwise }\end{cases}
\end{aligned}
$$

where $\gamma(k-1)$ and $\boldsymbol{e}_{1}(k)$ are obtained using (50) and (29), respectively.

For the nonlinear controller-driven model, its parameter estimation is obtained as follows:

$\hat{\boldsymbol{\Theta}}_{2}(k)=\operatorname{proj}\left\{\hat{\boldsymbol{\Theta}}_{2}^{\prime}(k)\right\}=\left\{\begin{array}{l}\hat{\boldsymbol{\Theta}}_{2}^{\prime}(k), \operatorname{det}\left[\hat{\boldsymbol{H}}_{0}(k)\right] \neq 0 \\ {\left[\ldots, \hat{\mathbf{H}}_{0}(k-1), \ldots\right]^{T}, \quad \text { otherwise }}\end{array}\right.$

where $\hat{\boldsymbol{\Theta}}_{2}(k)$ is obtained in (41) and (42), therein $\boldsymbol{h}(k)$ is again calculated from

$$
\begin{aligned}
\boldsymbol{h}_{2}(k) & =\frac{a(k) \boldsymbol{x}(k-1)}{1+\boldsymbol{x}(k-1)^{T} \boldsymbol{x}(k-1)} \\
a(k) & = \begin{cases}1, & \left\|\boldsymbol{e}_{2}(k)\right\|>2 \xi \\
0, & \text { otherwise }\end{cases}
\end{aligned}
$$

where $\xi>0$ is a pre-specified upper bound of the estimation error for the virtual unmodeled dynamics. $\boldsymbol{e}_{2}(k)$ is obtained from (41).

Condition 3: For the switching mechanism, we have $M=$ $2 \gamma(k-1)$.

To prove the stability and convergence, the following lemmas will be first established.

Lemma 1: The identification algorithm (28), (29) has the following properties.

1) $\left\|\hat{\boldsymbol{\Theta}}_{1}(k)-\boldsymbol{\Theta}_{\|} \mid \leq\right\| \hat{\boldsymbol{\Theta}}_{1}(0)-\boldsymbol{\Theta} \|$.

2) $\lim _{k \rightarrow \infty} \sum_{k=1}^{N} \frac{\mu_{1}(k)\left[\left\|\boldsymbol{e}_{1}(k)\right\|^{2}-4 \gamma(k-1)^{2}\right]}{2\left[1+\boldsymbol{x}(k-1)^{\mathrm{T}} \boldsymbol{x}(k-1)\right]}<\infty$.

3) $\lim _{k \rightarrow \infty} \frac{\mu_{1}(k)\left[\left\|\boldsymbol{e}_{1}(k)\right\|^{2}-4 \gamma(k-1)^{2}\right]}{2\left[1+\boldsymbol{x}(k-1)^{1} \boldsymbol{x}(k-1)\right]}=0$.

As for the identification algorithm (31)-(41), the following properties hold.

1) $\left\|\hat{\boldsymbol{\Theta}}_{2}(k)-\boldsymbol{\Theta}\right\| \leq\left\|\hat{\boldsymbol{\Theta}}_{2}(0)-\boldsymbol{\Theta}\right\|$.

2) $\lim _{k \rightarrow \infty} \frac{a(k)\left[\left.\left\|e_{2}(k)\right\|\right|^{2}-4 \xi^{2}\right]}{2\left[1+x(k-1)^{\mathrm{T}} x(k-1)\right]}=0$.

Proof: Let $\tilde{\boldsymbol{\Theta}}(k)=\hat{\boldsymbol{\Theta}}(k)-\boldsymbol{\Theta}, i=1,2$. One can then select the following Lyapunov function:

$$
V(k)=\tilde{\boldsymbol{\Theta}}(k)^{T} \tilde{\boldsymbol{\Theta}}(k) .
$$

Using the similar formulation to those in [7] the above results can be proved. This is omitted here.

Since (7) is equivalent to (10), in the following we will use (10) to analyze the system performance.

Lemma 2: When the linear adaptive control algorithm (28), (29) is applied to (7), the input and output dynamical equation of the system is given by

$$
\begin{aligned}
{\left[\tilde{\boldsymbol{B}}\left(z^{-1}\right)\right.} & \left.+\tilde{\boldsymbol{Q}}^{*}\left(1-z^{-1}\right) \boldsymbol{A}\left(z^{-1}\right)\right] \boldsymbol{y}(k+1)=\tilde{\boldsymbol{B}}\left(z^{-1}\right) \boldsymbol{e}_{1}(k+1) \\
& +\tilde{\boldsymbol{B}}\left(z^{-1}\right) \boldsymbol{w}(k+1)+\tilde{\boldsymbol{Q}}^{*}\left(1-z^{-1}\right) \boldsymbol{v}(k) \\
\left\{\boldsymbol{B}\left(z^{-1}\right)\right. & \left.+\boldsymbol{A}\left(z^{-1}\right) \boldsymbol{Q}^{*}\left(1-z^{-1}\right)\right\} \boldsymbol{u}(k) \\
& =\boldsymbol{A}\left(z^{-1}\right) \boldsymbol{w}(k+1)+\boldsymbol{A}\left(z^{-1}\right) \boldsymbol{e}_{1}(k+1)-\boldsymbol{v}(k) .
\end{aligned}
$$

Moreover, when the estimation algorithm for the virtual unmodeled dynamics (31)-(37) and the nonlinear adaptive control algorithm (40), (41) are applied to (10), the system input and output satisfy the following dynamics:

$$
\begin{aligned}
{\left[\tilde{\boldsymbol{B}}\left(z^{-1}\right)+\tilde{\boldsymbol{Q}}^{*}\left(1-z^{-1}\right) \boldsymbol{A}\left(z^{-1}\right)\right] \boldsymbol{y}(k+1) } \\
=\tilde{\boldsymbol{B}}\left(z^{-1}\right) \boldsymbol{e}_{2}(k+1)+\tilde{\boldsymbol{B}}\left(z^{-1}\right) \boldsymbol{w}(k+1) \\
\quad+\tilde{\boldsymbol{Q}}^{*}\left(1-z^{-1}\right) \boldsymbol{v}(k)-\tilde{\boldsymbol{B}}\left(z^{-1}\right) \boldsymbol{K}^{*}\left(1-z^{-1}\right) \hat{\boldsymbol{v}}(k) \\
\left\{\boldsymbol{B}\left(z^{-1}\right)+\boldsymbol{A}\left(z^{-1}\right) \boldsymbol{Q}^{*}\left(1-z^{-1}\right)\right\} \boldsymbol{u}(k) \\
=\boldsymbol{A}\left(z^{-1}\right) \boldsymbol{w}(k+1)+\boldsymbol{A}\left(z^{-1}\right) \boldsymbol{e}_{2}(k+1) \\
\quad-\boldsymbol{v}(k)-\boldsymbol{A}\left(z^{-1}\right) \boldsymbol{K}^{*}\left(1-z^{-1}\right) \hat{\boldsymbol{v}}(k)
\end{aligned}
$$

where $\tilde{\boldsymbol{B}}\left(z^{-1}\right)$ and $\tilde{\boldsymbol{Q}}^{*}\left(1-z^{-1}\right)$ will be determined by the following equations:

$$
\begin{aligned}
\tilde{\boldsymbol{B}}\left(z^{-1}\right) \boldsymbol{Q}^{*}\left(1-z^{-1}\right) & =\tilde{\boldsymbol{Q}}^{*}\left(1-z^{-1}\right) \boldsymbol{B}\left(z^{-1}\right) \\
\operatorname{det} \tilde{\boldsymbol{B}}\left(z^{-1}\right) & =\operatorname{det} \boldsymbol{B}\left(z^{-1}\right) .
\end{aligned}
$$

Proof: From (29), (30), and (44), it can be obtained that

$$
\begin{aligned}
\boldsymbol{e}_{1}(k+1) & =\boldsymbol{y}(k+1)-\hat{\boldsymbol{\Theta}}_{1}(k)^{T} \boldsymbol{x}(k) \\
& =\boldsymbol{y}(k+1)-\boldsymbol{w}(k+1)+\boldsymbol{Q}^{*}\left(1-z^{-1}\right) \boldsymbol{u}(k)
\end{aligned}
$$

by selecting matrices $\tilde{\boldsymbol{B}}\left(z^{-1}\right)$ and $\tilde{\boldsymbol{Q}}^{*}\left(1-z^{-1}\right)$ to satisfy (61) and (62).

By left-multiplying (10) with $\tilde{\boldsymbol{Q}}^{*}\left(1-z^{-1}\right)$ and (63) with $\tilde{\boldsymbol{B}}\left(z^{-1}\right)$, combining the resulting equations, and using (61) to replace the relevant terms associated with $\boldsymbol{u}(k)$, one can readily obtain (57).

Moreover, by left-multiplying (63) with $\boldsymbol{A}\left(z^{-1}\right)$ and using (10) to eliminate $\boldsymbol{y}(k+1)$, one can obtain (58).

Therefore, using (41) and (46), it can be obtained that

$$
\begin{aligned}
\boldsymbol{e}_{2}(k+1)= & \boldsymbol{y}(k+1)-\hat{\boldsymbol{\Theta}}_{2}(k)^{T} \boldsymbol{x}(k)-\hat{\boldsymbol{v}}(k) \\
= & \boldsymbol{y}(k+1)-\boldsymbol{w}(k+1)+\boldsymbol{Q}^{*}\left(1-z^{-1}\right) \boldsymbol{u}(k) \\
& +\boldsymbol{K}^{*}\left(1-z^{-1}\right) \hat{\boldsymbol{v}}(k) .
\end{aligned}
$$

Using the similar formulation, (59) and (60) can also be proved.

In terms of the stability and convergence analysis, minimum the state space realization of (7) will be used in order to simplify the analysis.

Lemma 3: Consider the following time-invariant nonlinear system:

$$
A\left(z^{-1}\right) y(k+1)=B\left(z^{-1}\right) u(k)+v(k)
$$

where $\{u(k)\},\{y(k)\}$ are the input and output of the system, respectively, and $A\left(z^{-1}\right)=1+a_{1} z^{-1}+\cdots+a_{n_{a}} z^{-n_{a}}$

$$
B\left(z^{-1}\right)=b_{0}+b_{1} z^{-1}+\cdots+b_{n_{b}} z^{-n_{b}} .
$$

Assuming that $A\left(z^{-1}\right)$ is an asymptotically stable polynomial and $v(k)$ satisfies the following condition:

$$
|v(k)| \leq \gamma(k), \quad \forall k
$$


where $\gamma(k)$ is an upper bounded function of $v(k)$, which is defined as $\gamma(k)=\varepsilon_{3}\|\bar{x}(k)\|+\varepsilon_{4}$ for some constant $\varepsilon_{3}$ and $\varepsilon_{4}$ (with $0 \leq \varepsilon_{3}<1$ and $\varepsilon_{4}>0$ ) and

$$
\overline{\boldsymbol{x}}(k)=\left[y(k), \ldots, y\left(k-n_{a}+1\right), u(k), \ldots, u\left(k-n_{b}\right)\right]^{\mathrm{T}} .
$$

Then, if $\varepsilon_{3}$ satisfies the condition that $0 \leq \varepsilon_{3} \leq 1-\|\Lambda\|$, ( $\Lambda$ is a matrix whose definition will be given in the sequel), there exist constants $\bar{C}_{1}$ and $\bar{C}_{2}$ that are independent of $k$, so that for all $1 \leq k \leq \infty, 0<\bar{C}_{1}<\infty$, and $0<\bar{C}_{2}<\infty$, we have

$$
|y(k)| \leq \bar{C}_{1}+\bar{C}_{2} \max _{0 \leq \tau \leq k}|u(\tau)| .
$$

Proof: Without loss of generality, let

$$
\overline{\boldsymbol{x}}(k)=\left[\overline{\boldsymbol{x}}_{1}(k)^{\mathrm{T}}, \overline{\boldsymbol{x}}_{2}(k)^{\mathrm{T}}\right]^{\mathrm{T}}
$$

where

$\overline{\boldsymbol{x}}_{1}(k)=\left[\bar{x}_{1,1}(k), \ldots, \bar{x}_{1, n_{a}}(k)\right]^{\mathrm{T}}=\left[y(k), \ldots, y\left(k-n_{a}+1\right)\right]^{\mathrm{T}}$, $\overline{\boldsymbol{x}}_{2}(k)=\left[\bar{x}_{2,1}(k), \ldots, \bar{x}_{2, n_{b}+1}(k)\right]^{\mathrm{T}}=\left[u(k), \ldots, u\left(k-n_{b}\right)\right]^{\mathrm{T}}$.

Then, (65) can be expressed into the state space equation as a minimum realization of input-output form as follows:

$$
\overline{\boldsymbol{x}}_{1}(k+1)=\Lambda \overline{\boldsymbol{x}}_{1}(k)+\Pi(k) \bar{u}(k)+\Xi v(k)
$$

where

$\begin{aligned} & \Lambda=\left[\begin{array}{ccccc}-a_{1} & -a_{2} & \cdots & -a_{n_{a}-1} & -a_{n_{a}} \\ 1 & 0 & \cdots & 0 & 0 \\ \vdots & \vdots & \cdots & \vdots & \vdots \\ 0 & 0 & \cdots & 0 & 0 \\ 0 & 0 & \cdots & 1 & 0\end{array}\right]_{n_{a} \times n_{a}}, \\ & \Pi=\left[\begin{array}{c}1 \\ 0 \\ \vdots \\ 0\end{array}\right]_{\left(n_{a}+1\right) \times 1} \bar{u}(k)=B\left(z^{-1}\right) u(k), \Xi=\left[\begin{array}{c}1 \\ 0 \\ \vdots \\ 0\end{array}\right]_{\left(n_{a}+1\right) \times 1} .\end{aligned}$

From (67) and (65), it can be obtained that

$$
\begin{aligned}
|v(k)| & \leq \varepsilon_{3}|| \overline{\boldsymbol{x}}(k) \|+\varepsilon_{4} \\
& \leq \varepsilon_{3}\left\|\overline{\boldsymbol{x}}_{1}(k)\right\|+\varepsilon_{3}|| \overline{\boldsymbol{x}}_{2}(k) \|+\varepsilon_{4} .
\end{aligned}
$$

Therefore, it can be shown that

$$
\begin{aligned}
& || \Pi u(k)+\Xi v(k) \| \leq\left(\sum_{i=0}^{n_{b}}\left|b_{i}\right|\right) \max _{0 \leq \tau \leq k}|u(\tau)|+|v(k)| \\
& \leq\left(\sum_{i=0}^{n_{b}}\left|b_{i}\right|+\varepsilon_{3} \sqrt{\left(n_{b}+1\right)}\right) \max _{0 \leq \tau \leq k}|u(\tau)|+\varepsilon_{3}|| \overline{\boldsymbol{x}}_{1}(k)||+\varepsilon_{4} .
\end{aligned}
$$

From (68), we have

$$
\overline{\boldsymbol{x}}_{1}(k+1)=\Lambda^{k+1} \overline{\boldsymbol{x}}_{1}(0)+\sum_{i=0}^{k} \Lambda^{i}[\Pi u(k-i)+\Xi v(k-i)] .
$$

Therefore, it can be shown that

$$
\begin{aligned}
\left\|\overline{\boldsymbol{x}}_{1}(k+1)\right\| \leq & \left\|\Lambda^{k+1}|||| \overline{\boldsymbol{x}}_{1}(0)\right\|+\sum_{i=0}^{k}|| \Lambda^{i}||[|| \Pi|| \cdot|u(k-i)| \\
& +\|\Xi\| \cdot|v(k-i)|] \\
\leq & \left\|\Lambda^{k+1}|||| \overline{\boldsymbol{x}}_{1}(0)\right\|+\sum_{i=0}^{k}|| \Lambda^{i}||\left[\left(\sum_{i=0}^{n_{b}}\left|b_{i}\right|\right.\right. \\
& \left.+\varepsilon_{3} \sqrt{\left(n_{b}+1\right)}\right) \max _{0 \leq \tau \leq k}|u(\tau)| \\
& \left.+\varepsilon_{3}\left\|\overline{\boldsymbol{x}}_{1}(k-i)\right\|+\varepsilon_{4}\right]
\end{aligned}
$$

Note that $A\left(z^{-1}\right)$ is an asymptotically stable polynomial and the conclusion that

$$
\operatorname{det}(\lambda I-\Lambda)=\lambda^{n} A\left(\lambda^{-1}\right)
$$

where $\lambda(0<\lambda<1)$ is a root of $A\left(z^{-1}\right)$.

Thus, there exist that $0<\eta<1$ and $K(0<K<\infty)$, so that for all, the following inequality holds [24]:

$$
\left\|\Lambda^{k}\right\| \leq K \cdot \eta^{k}
$$

Therefore, it can be readily shown from (72) that

$$
\begin{aligned}
& \left\|\overline{\boldsymbol{x}}_{1}(k+1)\right\| \leq K \cdot \eta^{k+1}|| \overline{\boldsymbol{x}}_{1}(0) \| \\
& \quad+\sum_{i=0}^{k} K \cdot \eta^{i}\left[\left(\sum_{i=0}^{n_{b}}\left|b_{i}\right|+\varepsilon_{3} \sqrt{\left(n_{b}+1\right)}\right) \max _{0 \leq \tau \leq k}|u(\tau)|\right. \\
& \left.\quad+\varepsilon_{3}\left\|\overline{\boldsymbol{x}}_{1}(k-i)\right\|+\varepsilon_{4}\right] .
\end{aligned}
$$

On the other hand, from (70) we can obtain that

$$
\begin{aligned}
\left\|\overline{\boldsymbol{x}}_{1}(k)\right\| \leq & \left(\|\Lambda\|+\varepsilon_{3}\right)\left\|\overline{\boldsymbol{x}}_{1}(k-1)\right\| \\
& +\left(\sum_{i=0}^{n_{b}}\left|b_{i}\right|+\varepsilon_{3} \sqrt{\left(n_{b}+1\right)}\right) \max _{0 \leq \tau \leq k}|u(\tau)|+\varepsilon_{4} .
\end{aligned}
$$

By recursively using the above inequality for $x(k-1)$, it can be obtained that

$$
\begin{aligned}
\left\|\overline{\boldsymbol{x}}_{1}(k)\right\| \leq & \left(\|\Lambda\|+\varepsilon_{3}\right)\left\|\overline{\boldsymbol{x}}_{1}(k-1)\right\| \\
& +\left(\sum_{i=0}^{n_{b}}\left|b_{i}\right|+\varepsilon_{3} \sqrt{\left(n_{b}+1\right)}\right) \max _{0 \leq \tau \leq k}|u(\tau)|+\varepsilon_{4} .
\end{aligned}
$$

It can be further shown that

$$
\begin{aligned}
\mid \overline{\boldsymbol{x}}_{1}(k) \| \leq & \left(\|\Lambda\|+\varepsilon_{3}\right)^{k}|| \overline{\boldsymbol{x}}_{1}(0) \|+\frac{1-\left(\|\Lambda\|+\varepsilon_{3}\right)^{k}}{1-\left(\|\Lambda\|+\varepsilon_{3}\right)} \\
\cdot & {\left[\left(\sum_{i=0}^{n_{b}}\left|b_{i}\right|+\varepsilon_{3} \sqrt{\left(n_{b}+1\right)}\right) \max _{0 \leq \tau \leq k}|u(\tau)|+\varepsilon_{4}\right] . }
\end{aligned}
$$

For the above inequality, when $\varepsilon_{3}$ satisfies the following condition:

$$
0 \leq \varepsilon_{3} \leq 1-\|\Lambda\|
$$


there exist $\overline{\bar{C}}_{1}>0$ and $\overline{\bar{C}}_{2}>0$ such that the following inequality holds:

$$
\begin{gathered}
\left(\|\Lambda\|+\varepsilon_{3}\right)^{k}|| \overline{\boldsymbol{x}}_{1}(0) \|+\frac{1-\left(\|\Lambda\|+\varepsilon_{3}\right)^{k}}{1-\left(\|\Lambda\|+\varepsilon_{3}\right)} \\
{\left[\sum_{i=0}^{n_{b}}\left|b_{i}\right|+\varepsilon_{4}\right] \leq \overline{\bar{C}}_{1}} \\
\frac{1-\left(\|\Lambda\|+\varepsilon_{3}\right)^{k}}{1-\left(\|\Lambda\|+\varepsilon_{3}\right)} \varepsilon_{3} \sqrt{\left(n_{b}+1\right)} \leq \overline{\bar{C}}_{2} .
\end{gathered}
$$

Therefore it can be seen that

$$
\left\|\overline{\boldsymbol{x}}_{1}(k)\right\| \leq \overline{\bar{C}}_{1}+\overline{\bar{C}}_{2} \max _{0 \leq \tau \leq k}|u(\tau)| .
$$

Similarly, there exist $\overline{\bar{C}}_{3}>0$ and $\overline{\bar{C}}_{4}>0$ so that

$$
\begin{aligned}
|| \overline{\boldsymbol{x}}_{1}(k-i) \| & \leq \overline{\bar{C}}_{3}+\overline{\bar{C}}_{4} \max _{0 \leq \tau \leq k-i}|u(\tau)| \\
& \leq \overline{\bar{C}}_{3}+\overline{\bar{C}}_{4} \max _{0 \leq \tau \leq k}|u(\tau)| .
\end{aligned}
$$

From (74) and (79), it can be finally obtained that

$$
\begin{aligned}
& \left\|\overline{\boldsymbol{x}}_{1}(k+1)\right\| \\
& \leq K \cdot \eta^{k+1}\left\|\overline{\boldsymbol{x}}_{1}(0)\right\|+\sum_{i=0}^{k} K \cdot \eta^{i}\left(\varepsilon_{3} \overline{\bar{C}}_{3}+\varepsilon_{3} \varepsilon_{4}\right) \\
& \quad+\sum_{i=0}^{k} K \cdot \eta^{i}\left[\sum_{i=0}^{n_{b}}\left|b_{i}\right|+\varepsilon_{3} \sqrt{\left(n_{b}+1\right)}\right. \\
& \left.\quad+\varepsilon_{3} \overline{\bar{C}}_{4}\right] \max _{0 \leq \tau \leq k}|u(\tau)| .
\end{aligned}
$$

Let

$$
\begin{gathered}
K \cdot \eta^{k+1}\left\|\overline{\boldsymbol{x}}_{1}(0)\right\|+\sum_{i=0}^{k} K \cdot \eta^{i}\left(\varepsilon_{3} \overline{\bar{C}}_{3}+\varepsilon_{3} \varepsilon_{4}\right) \leq \bar{C}_{1}, \\
\sum_{i=0}^{k} K \cdot \eta^{i}\left[\sum_{i=0}^{n_{b}}\left|b_{i}\right|+\varepsilon_{3} \sqrt{\left(n_{b}+1\right)}+\varepsilon_{3} \overline{\bar{C}}_{4}\right] \leq \bar{C}_{2} .
\end{gathered}
$$

Then, we can obtain that

$$
|y(k)| \leq\left\|\bar{x}_{1}(k)\right\| \leq \bar{C}_{1}+\bar{C}_{2} \max _{0 \leq \tau \leq k}|u(\tau)| .
$$

Using the above three lemmas, the following theorem can be proved.

Theorem 1: Assuming that (7) satisfies Conditions 1)-3) and the parameter matrices in polynomial matrices $\boldsymbol{A}\left(z^{-1}\right), \boldsymbol{B}\left(z^{-1}\right)$ are inside a compact set $\Omega$, then when the proposed adaptive control algorithm [i.e., the linear adaptive control algorithms (28), (29), the estimation algorithm for virtual unmodeled dynamics (31)-(37), the nonlinear adaptive control algorithm (40), (41) together with the switching mechanism (47) and (48)] is applied to (7), the closed-loop system is bounded-input-bounded-output (BIBO) stable. Moreover, when $k \rightarrow \infty$, the generalized tracking error converges to a small neighborhood of the origin, that is

$$
\left\|\overline{\boldsymbol{e}}_{g}(k+1)\right\| \leq \delta
$$

where

$$
\begin{aligned}
\overline{\boldsymbol{e}}_{g}(k+1):= & \boldsymbol{y}(k+1)-\left[\boldsymbol{w}(k+1)-\boldsymbol{Q}^{*}\left(1-z^{-1}\right) \boldsymbol{u}(k)\right. \\
& \left.-\boldsymbol{K}^{*}\left(1-z^{-1}\right) \boldsymbol{v}(k)\right]
\end{aligned}
$$

and $\delta=4\left\|\boldsymbol{K}^{*}\right\| \xi$ is a arbitrarily small positive number, $\xi$ is a pre-specified upper error bound of the compact set.

Proof: First we need to prove that the closed-loop switching can ensure the uniform boundedness of the input and output signals. For this purpose, two polynomial matrices $\left\{\tilde{\tilde{\boldsymbol{A}}}\left(z^{-1}\right), \tilde{\tilde{\boldsymbol{B}}}\left(z^{-1}\right)\right\}$ can selected so that the following equalities hold:

$$
\begin{aligned}
\boldsymbol{A}\left(z^{-1}\right) \tilde{\tilde{\boldsymbol{B}}}\left(z^{-1}\right) & =\boldsymbol{B}\left(z^{-1}\right) \tilde{\tilde{\boldsymbol{A}}}\left(z^{-1}\right) \\
\operatorname{det} \tilde{\tilde{\boldsymbol{B}}}\left(z^{-1}\right) & =\operatorname{det} \boldsymbol{B}\left(z^{-1}\right) .
\end{aligned}
$$

From (63) and (81) it can be seen that

$$
\operatorname{det} \tilde{\tilde{\boldsymbol{B}}}\left(z^{-1}\right)=\operatorname{det} \tilde{\boldsymbol{B}}\left(z^{-1}\right)=\operatorname{det} \boldsymbol{B}\left(z^{-1}\right) .
$$

Therefore using (62) and (80)-(82) together with the following equalities:

$$
\begin{aligned}
\operatorname{det} \boldsymbol{M} \boldsymbol{N} & =\operatorname{det} \boldsymbol{M} \operatorname{det} \boldsymbol{N}, \\
\operatorname{det} \boldsymbol{M}^{-1} & =1 / \operatorname{det} \boldsymbol{M}
\end{aligned}
$$

where $\boldsymbol{M}$ and $\boldsymbol{N}$ are any matrices of appropriate dimensions, it can be shown that

$$
\begin{aligned}
& \operatorname{det}\left[\tilde{\boldsymbol{B}}\left(z^{-1}\right)+\tilde{\boldsymbol{Q}}^{*}\left(1-z^{-1}\right) \boldsymbol{A}\left(z^{-1}\right)\right] \\
& =\operatorname{det}\left\{\tilde{\boldsymbol{B}}\left(z^{-1}\right)\left[\boldsymbol{I}+\tilde{\boldsymbol{B}}^{-1}\left(z^{-1}\right) \tilde{\boldsymbol{Q}}^{*}\left(1-z^{-1}\right) \boldsymbol{A}\left(z^{-1}\right)\right]\right\} \\
& =\operatorname{det}\left\{\tilde { \boldsymbol { B } } ( z ^ { - 1 } ) \left[\boldsymbol{I}+\tilde{\boldsymbol{B}}^{-1}\left(z^{-1}\right) \tilde{\boldsymbol{B}}\left(z^{-1}\right) \boldsymbol{Q}^{*}\left(1-z^{-1}\right)\right.\right. \\
& \left.\left.\left.\boldsymbol{B}^{-1}\left(z^{-1}\right) \boldsymbol{A}_{(} z^{-1}\right)\right]\right\} \\
& =\operatorname{det}\left\{\tilde{\boldsymbol{B}}\left(z^{-1}\right)\left[\boldsymbol{I}+\boldsymbol{Q}^{*}\left(1-z^{-1}\right) \boldsymbol{B}^{-1}\left(z^{-1}\right) \boldsymbol{A}\left(z^{-1}\right)\right]\right\} \\
& =\operatorname{det}\left\{\tilde { \boldsymbol { B } } ( z ^ { - 1 } ) \left[\boldsymbol{I}+\boldsymbol{Q}^{*}\left(1-z^{-1}\right) \boldsymbol{B}^{-1}\left(z^{-1}\right) \boldsymbol{B}\left(z^{-1}\right)\right.\right. \\
& \left.\left.\cdot \tilde{\tilde{\boldsymbol{A}}}\left(z^{-1}\right) \tilde{\tilde{\boldsymbol{B}}}^{-1}\left(z^{-1}\right)\right]\right\} \\
& =\operatorname{det}\left\{\tilde{\boldsymbol{B}}\left(z^{-1}\right)\left[\boldsymbol{I}+\boldsymbol{Q}^{*}\left(1-z^{-1}\right) \tilde{\tilde{\boldsymbol{A}}}\left(z^{-1}\right) \tilde{\tilde{\boldsymbol{B}}}^{-1}\left(z^{-1}\right)\right]\right\}
\end{aligned}
$$

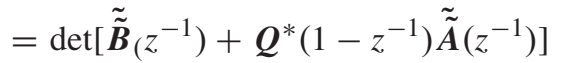

$$
\begin{aligned}
& =\operatorname{det}\left\{\left[\boldsymbol{A}^{-1}\left(z^{-1}\right) \boldsymbol{B}\left(z^{-1}\right)+\boldsymbol{Q}^{*}\left(1-z^{-1}\right)\right] \tilde{\tilde{\boldsymbol{A}}}\left(z^{-1}\right)\right\} \\
& =\operatorname{det}\left\{\boldsymbol{B}\left(z^{-1}\right)+\boldsymbol{A}\left(z^{-1}\right) \boldsymbol{Q}^{*}\left(1-z^{-1}\right)\right\} .
\end{aligned}
$$

From (13) it can then be concluded that the matrix $\boldsymbol{B}\left(z^{-1}\right)+$ $\boldsymbol{A}\left(z^{-1}\right) \boldsymbol{Q}^{*}\left(1-z^{-1}\right)$ is stable. Because $\boldsymbol{w}(k+1)$ is bounded and $\boldsymbol{v}(k)$ is the nonlinear disturbance satisfying Condition 1, using Lemma 3 together with (83) and (13), one can show that there exist positive constants $C_{1}, C_{2}$ so that

$$
\|\boldsymbol{y}(k)\| \leq C_{1}+C_{2} \max _{0 \leq \tau \leq k}\left\|\boldsymbol{e}_{1}(\tau)\right\| .
$$

Similarly, again using Condition 1 and Lemma 3 , together with (83) and (13) as well as (58) of Lemma 3, it can be seen that there exist positive constants $C_{3}, C_{4}$ so that

$$
\|\boldsymbol{u}(k-1)\| \leq C_{3}+C_{4} \max _{0 \leq \tau \leq k}\left\|\boldsymbol{e}_{1}(\tau)\right\| .
$$

From the structure of $\boldsymbol{x}(k-1)$ it can be seen that there exist positive constants $C_{5}, C_{6}$ so that

$$
\|\boldsymbol{x}(k-1)\| \leq C_{5}+C_{6} \max _{0 \leq \tau \leq k}\left\|\boldsymbol{e}_{1}(\tau)\right\| .
$$

Also, using (59), (60) of Lemma 2 and (83), (13) of Lemma 3 and following similar steps, it can be seen that there exist 
positive constants $C_{7}, C_{8}$ so that the following inequality is satisfied:

$$
\|\boldsymbol{x}(k-1)\| \leq C_{7}+C_{8} \max _{0 \leq \tau \leq k}\left\|\boldsymbol{e}_{2}(\tau)\right\| .
$$

In the following, we will prove that $\boldsymbol{x}(k-1)$ is bounded for the switching system.

Case 1: If $\left\|\boldsymbol{e}_{1}(k)\right\|>2 \gamma(k-1)$ and $\left\|\boldsymbol{e}_{2}(k)\right\|>2 \gamma(k-1)$, then

$$
J_{j}(k)=\sum_{t=1}^{k} \frac{\left[\left\|\boldsymbol{e}_{j}(t)\right\|^{2}-4 \gamma(t-1)^{2}\right]}{2\left[1+\boldsymbol{x}(t-1)^{\mathrm{T}} \boldsymbol{x}(t-1)\right]} .
$$

In this case, if the linear controller based on linear controllerdriven model is applied to the plant, from 3) of Lemma 1 it can seen that

$$
\lim _{k \rightarrow \infty} \frac{\left(\left\|\boldsymbol{e}_{1}(k)\right\|^{2}-4 \gamma(k-1)^{2}\right)}{2\left(1+\boldsymbol{x}(k-1)^{T} \boldsymbol{x}(k-1)\right)}=0 .
$$

Therefore, if $\left\{\boldsymbol{e}_{1}(k)\right\}$ is bounded, from (84) it can be concluded that $\boldsymbol{x}(k-1)$ is bounded. However, if $\left\{\boldsymbol{e}_{1}(k)\right\}$ is not bounded, there would be a sub-set of the sequence with index as $\left\{k_{n}\right\}$ so that

$$
\lim _{k_{n} \rightarrow \infty}\left\|\boldsymbol{e}_{1}\left(k_{n}\right)\right\|=\infty
$$

and $\left\|\boldsymbol{e}_{1}(k)\right\| \leq\left\|\boldsymbol{e}_{1}\left(k_{n}\right)\right\|$ for $k<k_{n}$. Along the index $\left\{k_{n}\right\}$, we have

$$
\begin{aligned}
& \frac{\left[\left\|\boldsymbol{e}_{1}\left(k_{n}\right)\right\|^{2}-4 \gamma\left(k_{n}-1\right)^{2}\right]}{2\left(1+\boldsymbol{x}\left(k_{n}-1\right)^{T} \boldsymbol{x}\left(k_{n}-1\right)\right)} \\
& \geq \frac{\left[\left\|\boldsymbol{e}_{1}\left(k_{n}\right)\right\|^{2}-4 \gamma\left(k_{n}-1\right)^{2}\right]}{2\left(1+\left(C_{5}+C_{6}\left\|\boldsymbol{e}_{1}\left(k_{n}\right)\right\|\right)^{2}\right)} \\
& \geq \frac{\left[\left\|\boldsymbol{e}_{1}\left(k_{n}\right)\right\|-2 \gamma\left(k_{n}-1\right)\right]^{2}}{2\left(1+\left(C_{5}+C_{6}\left\|\boldsymbol{e}_{1}\left(k_{n}\right)\right\|\right)^{2}\right)} \\
& \geq \frac{\left[\left\|\boldsymbol{e}_{1}\left(k_{n}\right)\right\|-2 \gamma\left(k_{n}-1\right)\right]}{\left[2\left(1+\left(C_{5}+C_{6}\left\|\boldsymbol{e}_{1}\left(k_{n}\right)\right\|\right)^{2}\right)\right]^{\frac{1}{2}}} \\
& =\frac{\left[\left\|\boldsymbol{e}_{1}\left(k_{n}\right)\right\|-2 \gamma\left(k_{n}-1\right)\right]}{\left[(\sqrt{2})^{2}+\left[\sqrt{2}\left(C_{5}+C_{6}\left\|\boldsymbol{e}_{1}\left(k_{n}\right)\right\|\right)\right]^{2}\right]^{\frac{1}{2}}} \\
& \geq \frac{\left[\left\|\boldsymbol{e}_{1}\left(k_{n}\right)\right\|-2 \gamma\left(k_{n}-1\right)\right]}{\sqrt{2}+\left[\sqrt{2}\left(C_{5}+C_{6}\left\|\boldsymbol{e}_{1}\left(k_{n}\right)\right\|\right)\right]} \\
& \geq \frac{1-2 \gamma\left(k_{n}-1\right) /\left\|\boldsymbol{e}_{1}\left(k_{n}\right)\right\|}{\sqrt{2} /\left\|\boldsymbol{e}_{1}\left(k_{n}\right)\right\|+\sqrt{2} C_{5} /\left\|\boldsymbol{e}_{1}\left(k_{n}\right)\right\|+\sqrt{2} C_{6}} .
\end{aligned}
$$

Also, since the following inequality holds:

$$
\begin{aligned}
\gamma\left(k_{n}-1\right) & =\varepsilon_{1}\left\|\boldsymbol{x}\left(k_{n}-1\right)\right\|+\varepsilon_{2} \\
& \leq \varepsilon_{1}\left[C_{5}+C_{6}\left\|\boldsymbol{e}_{1}\left(k_{n}\right)\right\|\right]+\varepsilon_{2} \\
& \left.=\varepsilon_{1} C_{5}+\varepsilon_{2}+\varepsilon_{1} C_{6}\left\|\boldsymbol{e}_{1}\left(k_{n}\right)\right\|\right]
\end{aligned}
$$

it can be seen that

$$
\begin{aligned}
& \frac{\left[\left\|\boldsymbol{e}_{1}\left(k_{n}\right)\right\|^{2}-4 \gamma\left(k_{n}-1\right)^{2}\right]}{2\left(1+\boldsymbol{x}\left(k_{n}-1\right)^{T} \boldsymbol{x}\left(k_{n}-1\right)\right)} \\
& \quad \geq \frac{1-2 \varepsilon_{1} C_{6}-2\left[\varepsilon_{1} C_{5}+\varepsilon_{2}\right] /\left\|\boldsymbol{e}_{1}\left(k_{n}\right)\right\|}{\sqrt{2} /\left\|\boldsymbol{e}_{1}\left(k_{n}\right)\right\|+\sqrt{2} C_{5} /\left\|\boldsymbol{e}_{1}\left(k_{n}\right)\right\|+\sqrt{2} C_{6}} .
\end{aligned}
$$

Therefore, there exists $\varepsilon_{1}$ so that the following holds:

$$
\lim _{k_{n} \rightarrow \infty} \frac{\left[\left\|\boldsymbol{e}_{1}\left(k_{n}\right)\right\|^{2}-4 \gamma\left(k_{n}-1\right)^{2}\right]}{2\left(1+\boldsymbol{x}\left(k_{n}-1\right)^{T} \boldsymbol{x}\left(k_{n}-1\right)\right)} \geq \frac{1-2 \varepsilon_{1} C_{6}}{\sqrt{2} C_{6}} \neq 0 .
$$

This contradicts (87) and indicates that the assumption that $\left\{\boldsymbol{e}_{1}(k)\right\}$ is unbounded is not correct. Therefore, $\left\{\boldsymbol{e}_{1}(k)\right\}$ is bounded and $\boldsymbol{x}(k-1)$ is also bounded. As a result, the input and output signals of the closed-loop system are bounded.

When the nonlinear controller based on nonlinear controllerdriven model is applied to the system, using 2) of Lemma 1, it can be seen that

$$
\lim _{k \rightarrow \infty} \frac{\left[\left\|\boldsymbol{e}_{2}(k)\right\|^{2}-4 \xi^{2}\right]}{2\left[1+\boldsymbol{x}(k-1)^{\mathrm{T}} \boldsymbol{x}(k-1)\right]}=0 .
$$

Assuming again that $\left\{\boldsymbol{e}_{2}(k)\right\}$ is an unbounded sequence, we can use the similar formulation to the above to obtain the following result:

$$
\lim _{k \rightarrow \infty} \frac{\left[\left\|\boldsymbol{e}_{2}\left(k_{n}\right)\right\|^{2}-4 \xi^{2}\right]}{2\left[1+\boldsymbol{x}\left(k_{n}-1\right)^{\mathrm{T}} \boldsymbol{x}\left(k_{n}-1\right)\right]}=\frac{1}{\sqrt{2} C_{8}}>0 .
$$

This has shown a contradiction to 2). Therefore $\left\{\boldsymbol{e}_{2}(k)\right\}$ should be bounded. This means that $\boldsymbol{x}(k-1)$ is bounded and the input and output signals of the system are uniformly bounded. This indicates that the closed-loop switching system is BIBO stable.

Case 2: If $\left\|\boldsymbol{e}_{1}(k)\right\| \leq 2 \gamma(k-1)$ or $\left\|\boldsymbol{e}_{2}(k)\right\| \leq 2 \gamma(k-1)$, then

$$
J_{j}(k)=c\left[1-\mu_{j}(t)\right]\left\|\boldsymbol{e}_{j}(t)\right\|^{2} .
$$

In this case, we need to analyze the estimation errors $\boldsymbol{e}_{1}(k+1)$ and $\boldsymbol{e}_{2}(k+1)$.

For this purpose, from (26), (29), (30), (43), and (44), it can be seen that

$$
\begin{aligned}
\boldsymbol{e}_{1}(k+1) & =\boldsymbol{y}(k+1)-\hat{\boldsymbol{\Theta}}_{1}(k)^{T} \boldsymbol{x}(k) \\
& =\boldsymbol{y}(k+1)-\left[\boldsymbol{w}(k+1)-\boldsymbol{Q}^{*}\left(1-z^{-1}\right) \boldsymbol{u}(k)\right] .
\end{aligned}
$$

Therefore, when $k \rightarrow \infty$, from the identification algorithm given by (51)-(53) and (96) it can be seen that $\boldsymbol{e}_{1}(k+1)$ should satisfy

$$
v(k) \leq\left\|\boldsymbol{e}_{1}(k+1)\right\| \leq 2 \gamma(k) .
$$

From (39), (41), (42), (45), and (46), we have

$$
\begin{aligned}
\boldsymbol{e}_{2}(k+1)= & \boldsymbol{y}(k+1)-\hat{v}(k)-\hat{\boldsymbol{\Theta}}_{2}(k)^{T} \boldsymbol{x}(k) \\
= & \boldsymbol{y}(k+1)-\left[\boldsymbol{w}(k+1)-\boldsymbol{Q}^{*}\left(1-z^{-1}\right) \boldsymbol{u}(k)\right. \\
& \left.-\boldsymbol{K}^{*}\left(1-z^{-1}\right) \hat{\boldsymbol{v}}(k)\right] \\
= & \boldsymbol{y}(k+1)-[\boldsymbol{\Theta} \boldsymbol{x}(k)+\boldsymbol{v}(k) \\
& \left.+\boldsymbol{K}^{*}\left(1-z^{-1}\right) \boldsymbol{v}(k)\right]+\boldsymbol{K}^{*}\left(1-z^{-1}\right) \hat{\boldsymbol{v}}(k) \\
= & \boldsymbol{K}^{*}\left(1-z^{-1}\right) \hat{\boldsymbol{v}}(k)-\boldsymbol{K}^{*}\left(1-z^{-1}\right) \boldsymbol{v}(k) \\
= & \boldsymbol{K}^{*}\left(1-z^{-1}\right)[\overline{\boldsymbol{v}}(k)-\boldsymbol{v}(k)] \\
& +\boldsymbol{K}^{*}\left(1-z^{-1}\right)[\hat{\boldsymbol{v}}(k)-\overline{\boldsymbol{v}}(k)] .
\end{aligned}
$$

Therefore it can be seen that

$$
\begin{aligned}
\left\|\boldsymbol{e}_{2}(k+1)\right\| \leq & \left\|\boldsymbol{K}^{*}\left(1-z^{-1}\right)\right\|\|\overline{\boldsymbol{v}}(k)-\boldsymbol{v}(k)\| \\
& +\left\|\boldsymbol { K } ^ { * } \left|\|\mid \hat{\boldsymbol{v}}(k)-\overline{\boldsymbol{v}}(k)\|+\left\|\boldsymbol{K}^{*}\right\| \| \hat{\boldsymbol{v}}(k-1)\right.\right. \\
& -\overline{\boldsymbol{v}}(k-1) \| .
\end{aligned}
$$


Since when $k \rightarrow \infty,\|\overline{\boldsymbol{v}}(k)-\boldsymbol{v}(k)\|$ is bounded by a small constant, it can be concluded that

$$
\begin{aligned}
\left\|\boldsymbol{e}_{2}(k+1)\right\| \leq & \left\|\boldsymbol{K}^{*}\right\|\|\hat{\boldsymbol{v}}(k)-\overline{\boldsymbol{v}}(k)\| \\
& +\left\|\boldsymbol{K}^{*}\right\|\|\hat{\boldsymbol{v}}(k-1)-\overline{\boldsymbol{v}}(k-1)\| \\
\leq & 2\left\|\boldsymbol{K}^{*}\right\| \xi .
\end{aligned}
$$

At this stage, from (97) and (47), (48), (49) as well as (99), we have

$$
\begin{aligned}
J_{1}(k) & =c\left\|\boldsymbol{e}_{1}(t)\right\|^{2} \\
& \geq c\|v(k)\|^{2} \\
J_{2}(k) & =c\left\|\boldsymbol{e}_{2}(t)\right\|^{2} \\
& \leq 2 c\left\|\boldsymbol{K}^{*}\right\| \xi .
\end{aligned}
$$

This indicates that there exists a sample time $K$ so that when $k>K$, we have

$$
J_{2}(k)<J_{1}(k) .
$$

This means that after a finite period of time the nonlinear controller based on nonlinear controller-driven model will drive the system. Using (85) and (99) it can be again concluded that $\boldsymbol{x}(k-1)$ is bounded and the closed-loop system is BIBO stable.

From the above analyses, it can be concluded that the input and output signals for the closed-loop system are uniformly bounded no matter which adaptive controller is applied to the system. In the following, we will analyze the convergence of the proposed algorithm. From (64), it can be seen that

$$
\begin{aligned}
\overline{\boldsymbol{e}}_{g}(k+1)= & \boldsymbol{e}_{2}(k+1)+\boldsymbol{K}^{*}\left(1-z^{-1}\right)[\boldsymbol{v}(k)-\hat{\boldsymbol{v}}(k)] \\
= & \boldsymbol{e}_{2}(k+1)+\boldsymbol{K}^{*}\left(1-z^{-1}\right)\{[\boldsymbol{v}(k)-\overline{\boldsymbol{v}}(k)] \\
& +[\overline{\boldsymbol{v}}(k)-\hat{\boldsymbol{v}}(k)]\} \\
= & \boldsymbol{e}_{2}(k+1)+\boldsymbol{K}^{*}\left(1-z^{-1}\right)[\boldsymbol{v}(k)-\overline{\boldsymbol{v}}(k)] \\
& +\boldsymbol{K}^{*}\left(1-z^{-1}\right)[\overline{\boldsymbol{v}}(k)-\hat{\boldsymbol{v}}(k)] .
\end{aligned}
$$

When $k \rightarrow \infty$, from (98) and (99) it can be shown that

$$
\left\|\overline{\boldsymbol{e}}_{g}(k+1)\right\| \leq 4\left\|\boldsymbol{K}^{*}\right\| \xi=\delta
$$

where $\delta=4\left\|\boldsymbol{K}^{*}\right\| \xi$. From (38) it can be concluded that there exists a desired fuzzy system so that $\xi$ can be made arbitrarily small. Therefore, the number $\delta$ can also be made very small. This indicates that the generalized tracking error of the closedloop system converges to a small neighborhood of the origin.

Remark 3: Note that the results of Theorem 1 are certainly not global. However, since complex industrial systems often work near their operating points, local results are often expected and most useful.

\section{ViI. Simulation and Test on a Twin-Tank Level CONTROL SySTEM}

\section{A. Simulation Study}

In this section, we will demonstrate the ability of the proposed method for the effective control of three classes of two-inputs and two-outputs nonlinear discrete time systems with unstable zero dynamics. The systems are assumed to have either known or unknown model structure and unknown parameters, and are subject to either random bounded disturbances or unknown bounded disturbance. The following three simulation tests are carried out.

1) Nonlinear System Model $\Sigma 1$ : We consider the following system:

$$
\begin{aligned}
y_{1}(k+1)= & 0.3 y_{1}(k)+0.7 u_{1}(k)+0.6 u_{1}(k-1) \\
& +0.5 u_{2}(k)+0.3 u_{2}(k-1) \\
& +0.2 y_{2}(k-1) y_{1}(k) /\left[1+y_{2}^{2}(k-1)+y_{1}^{2}(k)\right] \\
& +0.3 u_{1}(k) \sin \left[u_{1}(k-1) u_{2}(k)\right] \\
y_{2}(k+1)= & -0.1 y_{2}(k)+0.7 u_{1}(k)+0.4 u_{1}(k-1) \\
& +0.8 u_{2}(k)+u_{2}(k-1) \\
& +0.2 y_{2}(k) u_{1}(k) /\left[1+u_{1}^{2}(k)+y_{2}^{2}(k)\right] \\
& +0.1 u_{2}(k) \sin \left(u_{2}^{2}(k-1)\right)-0.1 \sin \left[0.5 y_{2}^{2}(k-1)\right]
\end{aligned}
$$

where the nonlinearity is given by

$$
\begin{aligned}
& 0.2 y_{2}(k-1) y_{1}(k) /\left[1+y_{2}^{2}(k-1)+y_{1}^{2}(k)\right] \\
& +0.3 u_{1}(k) \sin \left[u_{1}(k-1) u_{2}(k)\right]
\end{aligned}
$$

and

$$
\begin{aligned}
& 0.2 y_{2}(k) u_{1}(k) /\left[1+u_{1}^{2}(k)+y_{2}^{2}(k)\right] \\
& +0.1 u_{2}(k) \sin \left(u_{2}^{2}(k-1)\right)-0.1 \sin \left[0.5 y_{2}^{2}(k-1)\right]
\end{aligned}
$$

which are not globally bounded.

From system $\Sigma 1$ it can be seen that

$$
B\left(z^{-1}\right)=\left[\begin{array}{cc}
0.7+0.6 z^{-1} & 0.5+0.3 z^{-1} \\
0.7+0.4 z^{-1} & 0.8+z^{-1}
\end{array}\right] .
$$

Since the roots of det $B\left(z^{-1}\right)=0.21+0.77 z^{-1}+0.48 z^{-2}$ are -2.8703 and -0.7963 , respectively, the zero dynamics of the above nonlinear system are unstable.

In this case, the structure orders of the linear controllerdriven model are selected to be the same as those of the plant, leading to $n_{h}=n_{b}=1, n_{g}=n_{a}-1=1$. Therefore, the parameters of controller-driven model have the following parameter matrices:

$$
\begin{aligned}
& \boldsymbol{G}\left(z^{-1}\right)=\left[\begin{array}{ll}
g_{11}^{0} & g_{12}^{0} \\
g_{21}^{0} & g_{22}^{0}
\end{array}\right] \\
& \boldsymbol{H}\left(z^{-1}\right)=\left[\begin{array}{ll}
h_{11}^{0}+h_{11}^{1} z^{-1} & h_{12}^{0}+h_{12}^{1} z^{-1} \\
h_{21}^{0}+h_{21}^{1} z^{-1} & h_{22}^{0}+h_{22}^{1} z^{-1}
\end{array}\right] .
\end{aligned}
$$

The data vector can therefore be expressed as

$$
\begin{aligned}
\boldsymbol{x}(k) & =\left[x_{1}(k), x_{2}(k), \ldots, x_{6}(k)\right]^{\mathrm{T}} \\
& =\left[y_{1}(k), y_{2}(k), u_{1}(k), u_{2}(k), u_{1}(k-1), u_{2}(k-1)\right]^{\mathrm{T}} .
\end{aligned}
$$

In this case, the $\boldsymbol{h}(k)$ in the identification algorithm (28) becomes

$$
\boldsymbol{h}(k)=\frac{\mu_{1}(k) \boldsymbol{x}(k-1)}{1+\boldsymbol{x}(k-1)^{T} \boldsymbol{x}(k-1)}
$$

where

$$
\begin{aligned}
\boldsymbol{x}(k-1)= & {\left[x_{1}(k-1), x_{2}(k-1), \ldots, x_{6}(k-1)\right]^{\mathrm{T}} } \\
= & {\left[y_{1}(k-1), y_{2}(k-1), u_{1}(k-1),\right.} \\
& \left.u_{2}(k-1), u_{1}(k-2), u_{2}(k-2)\right]^{\mathrm{T}} \\
\mu_{1}(k)= & \left\{\begin{array}{l}
1, \quad\left\|\boldsymbol{e}_{1}(k)\right\|>2 \gamma(k-1) \\
0, \text { otherwise. }
\end{array}\right.
\end{aligned}
$$



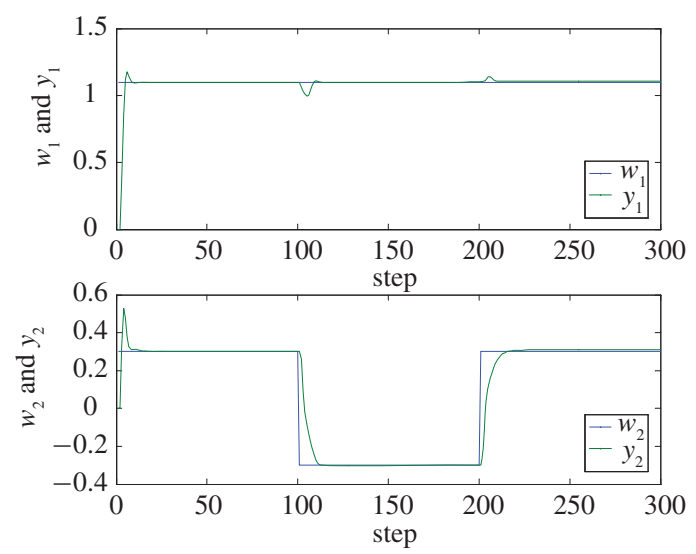

Fig. 4. Responses of system output $\boldsymbol{y}(k)$ and the desired output $\boldsymbol{w}(k)$ when the switching control is used.
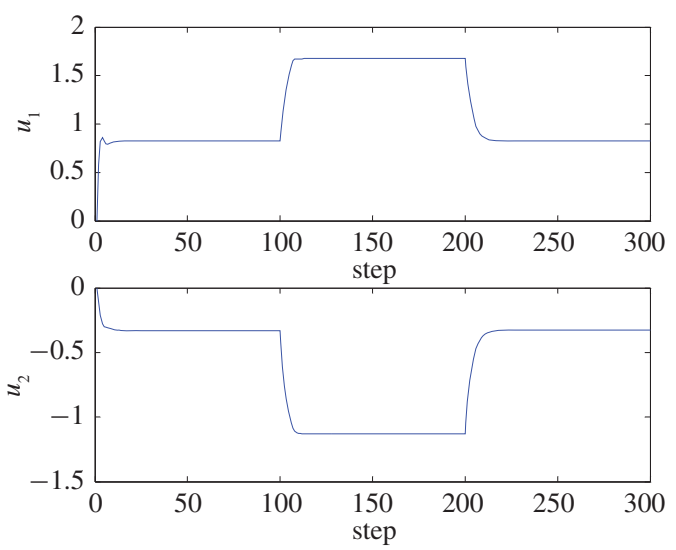

Fig. 5. Control input responses $u_{1}(k)$ and $u_{2}(k)$ when the switching control is used.

By selecting $\varepsilon_{1}$ and $\varepsilon_{2}$ as 0.0001 and 0.1 , respectively, we have

$$
\gamma(k-1)=0.0001\|x(k-1)\|+0.1 .
$$

Using (13), the weighting matrix $Q^{*}\left(1-z^{-1}\right)$ can be

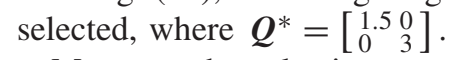

Moreover, by selecting

$$
\boldsymbol{K}^{*}\left(z^{-1}\right)=\operatorname{diag}\{0.3,0.5\}
$$

the eigenvalues of the closed-loop system are given by $0.7372,-0.1362$, and $0.2788 \pm 0.3003 i$ which are all inside the unit circle. From $(44), \boldsymbol{u}_{1}(k)$ can be readily calculated.

From (31), we have $\overline{\boldsymbol{v}}(k)$ and can then normalize $\overline{\boldsymbol{v}}(k)$ using (32). Algorithm (31)-(37) can then be used to calculate the estimated value for $\boldsymbol{v}(k)$ to give $\hat{\boldsymbol{v}}(k)$, where the membership functions of ANFIS are of the Gaussian type with two fuzzy sets for each input. Then identification algorithm (40) and (41) can be used to estimate the parameters of the controller-driven model to obtain $\hat{\boldsymbol{\Theta}}_{2}(k)$, where in $(41), \xi=10^{-4}$. This will lead to $\boldsymbol{u}_{2}(k)$ from the controller (46).

In the normalization of $\overline{\boldsymbol{v}}(k), \varepsilon_{1}$ and $\varepsilon_{2}$ are selected as 0.0001 and 0.1 , respectively.

For the switching function, the parameters are selected as $c=1$. Also, the initial states of the system are set to $y_{1}(0)=0, y_{2}(0)=0, u_{1}(0)=0$, and $u_{2}(0)=0$.

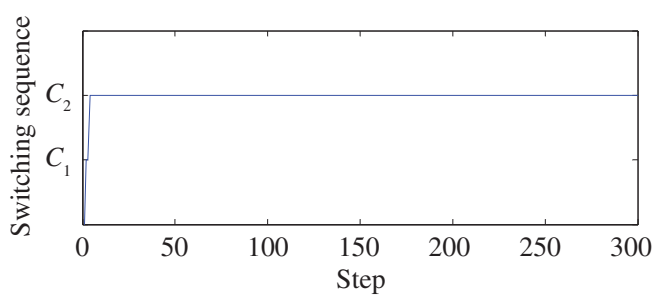

Fig. 6. Switching sequence.
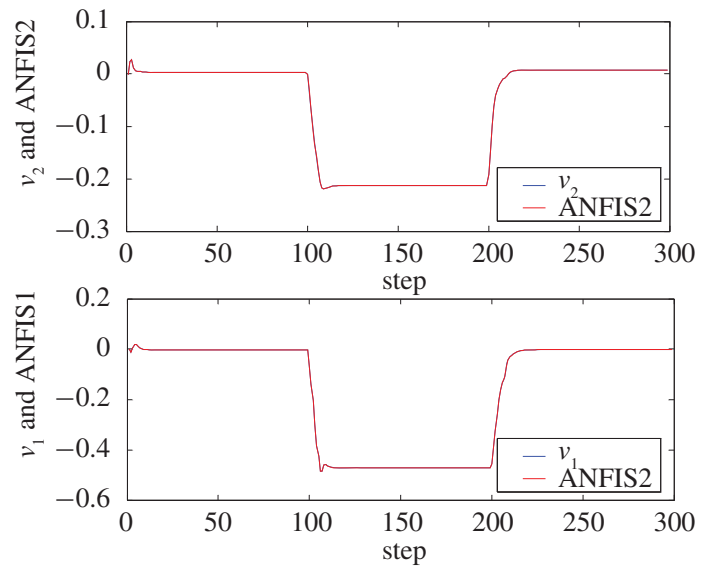

Fig. 7. Virtual unmodeled dynamics $\boldsymbol{v}(k)$ and its estimation by ANFIS.

In the simulations, the set-points for each sub-system are given as follows:

$$
\begin{aligned}
& w_{1}(k)=1.1 \\
& w_{2}(k)=0.3 \operatorname{sign}[\sin (\pi k / 100)] .
\end{aligned}
$$

Using the proposed adaptive switching control for the simulation tests for $\Sigma 1$, the results shown in Figs. 4-6 are obtained, where Fig. 4 displays the output of the closed-loop system and the desired output. Fig. 5 gives the control inputs $u_{1}(k)$ and $u_{2}(k)$ while Fig. 6 shows the switching sequence for the adaptive controller based on linear and nonlinear controllerdriven model.

From Figs. 4 and 5 it can be seen that since the initial states are zero, the identification does not produce accurate parameter estimation, the tracking performance of the closedloop system are not good. However, along with the improvement of the parameter estimation, the tracking performance of the closed-loop can be significantly improved through active compensation of the virtual unmodeled dynamics.

From the switching sequence in Fig. 6, it can be seen that initially the estimation error is big and accordingly the linear controller has been selected to stabilize the system. When $k=5$, the nonlinear control action is selected so as to improve the closed-loop performance.

Fig. 7 shows the estimation to the virtual unmodeled dynamics, where it can be seen that desired approximation results have been obtained when the estimation algorithm for the virtual unmodeled dynamics of this paper is used.

To illustrate the effectiveness of the proposed method in light of the results [5]-[9] as well as to show that ANFIS is better than BP neural networks for this application, the 

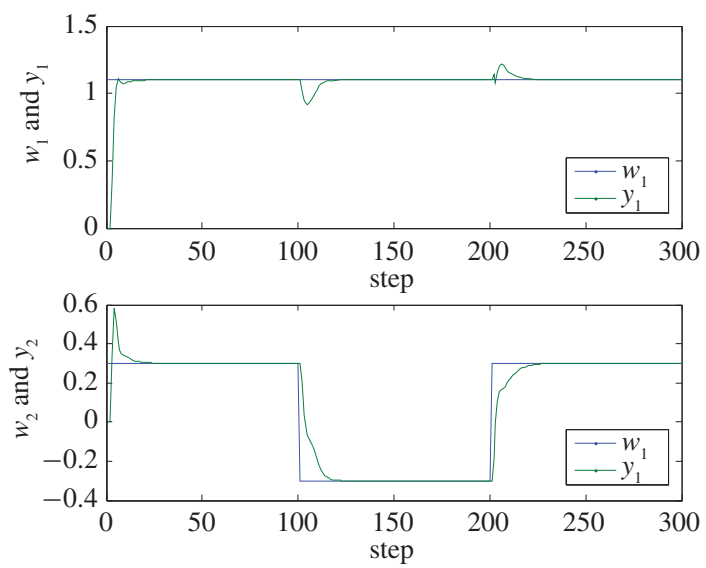

Fig. 8. Responses of system output $y(k)$ and the desired output $\boldsymbol{w}(k)$ when BP neural network is used.
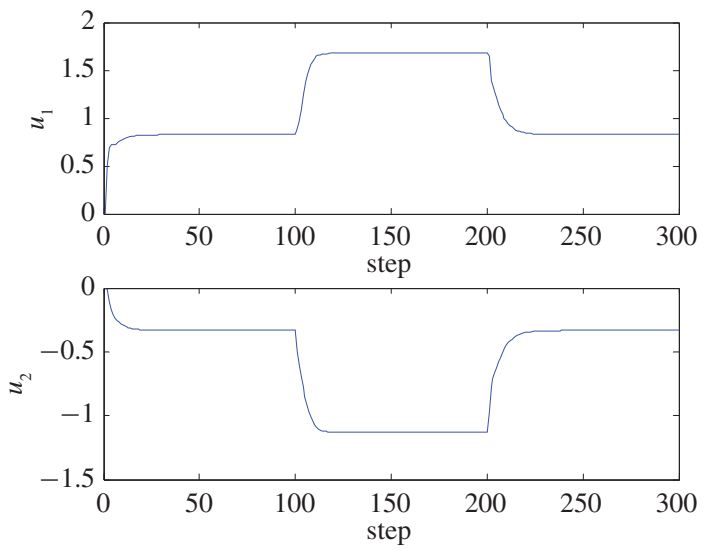

Fig. 9. Control input responses $u_{1}(k)$ and $u_{2}(k)$ when BP neural network is used.

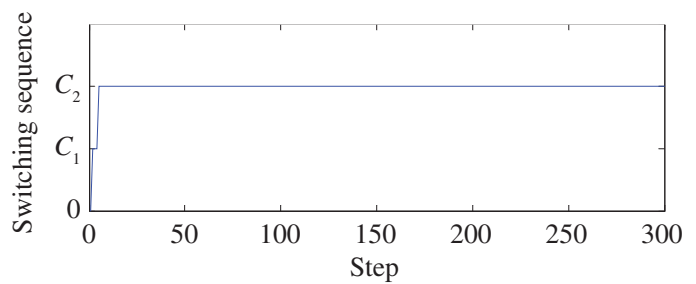

Fig. 10. Switching sequence.

results of the proposed method are compared with the results of [5]-[9]. ANFIS used in the proposed scheme is also compared with that with a BP neural network. The simulation results demonstrate the superior performance of the proposed approach over the results in [5]-[9]. However, due to the space limitation, in the following, we only show the comparison results with [7], which is the benchmark in this research area. In simulations, the two layer BP neural networks have been used to approach $\boldsymbol{v}(k)$. The node number of hidden layer in the network is selected as 18 , where the activation function is of the S-transfer function and the activation function of output layer is the linear transfer function. The learning rate is set to $l r=1$ and momentum factor is selected as $m c=0.9$. The performance of the switching system using the BP neural networks and multiple models is shown in Figs. 8-11.
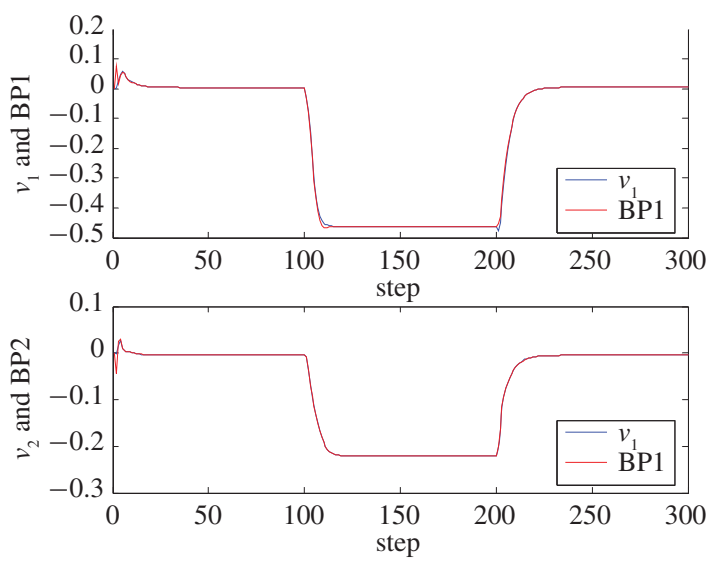

Fig. 11. Virtual unmodeled dynamics $\boldsymbol{v}(k)$ and its estimation by BP neural network.

By comparing Figs. 4-7 with Figs. 8-11, it can be concluded that the method proposed in this paper improves the control effects.

2) Nonlinear System Model $\Sigma 2$ : In this case, we use the following nonlinear system to carry out the simulation test:

$$
\begin{aligned}
y_{1}(k+1)= & 0.3 y_{1}(k)+0.7 u_{1}(k)+0.6 u_{1}(k-1) \\
& +0.5 u_{2}(k)+0.3 u_{2}(k-1) \\
& +0.2 y_{2}(k-1) y_{1}(k) /\left[1+y_{2}^{2}(k-1)+y_{1}^{2}(k)\right] \\
& +0.3 u_{1}(k) \sin \left[u_{1}(k-1) u_{2}(k)\right]+d_{1}(k) \\
y_{2}(k+1)= & -0.1 y_{2}(k)+0.7 u_{1}(k)+0.4 u_{1}(k-1) \\
& +0.8 u_{2}(k)+u_{2}(k-1) \\
& +0.2 y_{2}(k) u_{1}(k) /\left[1+u_{1}^{2}(k)+y_{2}^{2}(k)\right] \\
& +0.1 u_{2}(k) \sin \left(u_{2}^{2}(k-1)\right) \\
& -0.1 \sin \left[0.5 y_{2}^{2}(k-1)\right]+d_{2}(k)
\end{aligned}
$$

where $d_{1}(k)$ and $d_{2}(k)$ are unknown but bounded random disturbances with their upper bounds being given by 0.1 .

The model structure (orders $n_{g}$ and $n_{h}$ ) of the controllerdriven model, the data vectors $\boldsymbol{x}(k)$ and $\boldsymbol{h}(k)$ in the identification algorithm (28), the parameters $\varepsilon_{1}$ and $\varepsilon_{2}$ for the virtual unmodeled dynamics are the same as those in the first simulation example. Furthermore, $\gamma(k-1)$ in the estimation algorithm (29), the parameter $\{c\}$ in the switching function and the weighting matrices $\left\{\boldsymbol{Q}\left(z^{-1}\right), \boldsymbol{K}\left(z^{-1}\right)\right\}$ in the equation of the controller are the same as those in the first simulation example. The simulation results are shown in Figs. 12-14, where Fig. 12 shows the output of the closed-loop system and the desired output, Fig. 13 displays the responses of $u_{1}(k)$ and $u_{2}(k)$ and Fig. 14 gives the switching sequence for the linear and nonlinear adaptive controllers.

From Figs. 12 and 13 it can be seen that the proposed adaptive control algorithm can produce desired tracking performance for the closed-loop system even if the system is subjected to unknown and bounded random disturbances.

Fig. 15 shows the estimation results of the virtual unmodeled dynamics, where it can be seen that although the system is affected by the random disturbances that leads to 

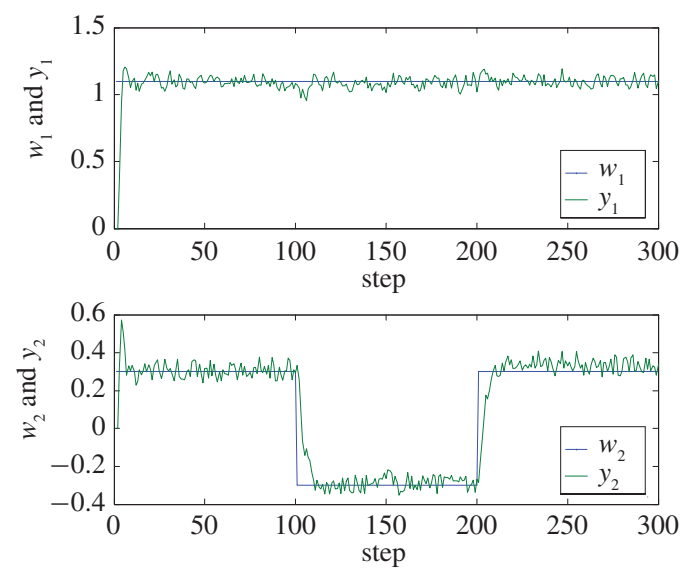

Fig. 12. Responses of the system and the desired outputs $\boldsymbol{y}(k)$ and $\boldsymbol{w}(k)$ when the switching controller is used.
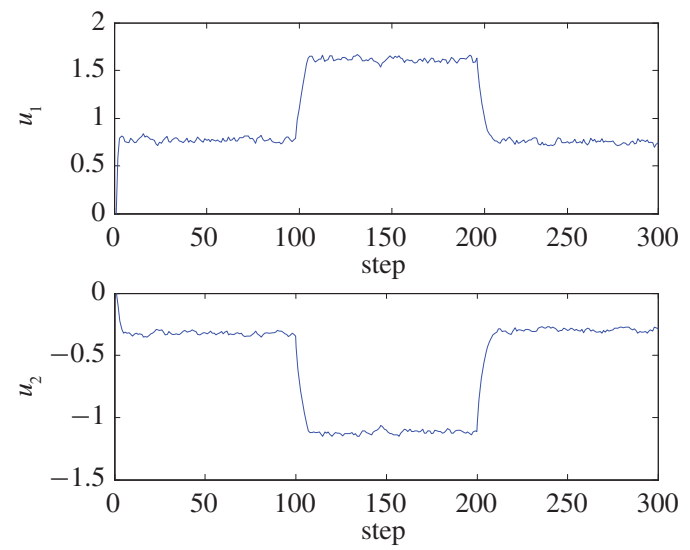

Fig. 13. Control inputs $u_{1}(k)$ and $u_{2}(k)$ when the switching controller is used.

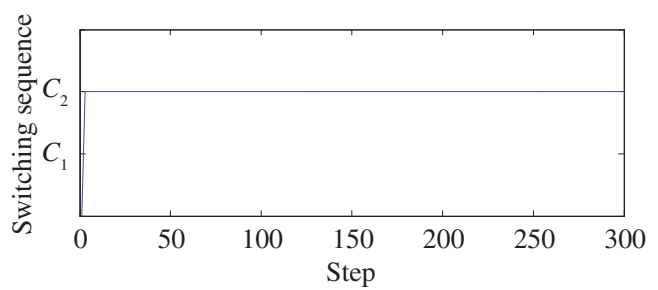

Fig. 14. Switching sequence.

certain errors in the approximation, the output of the closedloop system can still track the desired output reasonably well.

3) Nonlinear System Model $\Sigma$ 3: To further our simulation studies, we consider the following nonlinear system:

$$
\begin{aligned}
y_{1}(k+1)= & 0.3 y_{1}(k)+0.2 y_{1}(k-1)+0.7 u_{1}(k) \\
& +0.6 u_{1}(k-1)+0.5 u_{2}(k)+0.3 u_{2}(k-1) \\
& +0.2 y_{2}(k-1) y_{1}(k) /\left[1+y_{2}^{2}(k-1)+y_{1}^{2}(k)\right] \\
& +0.3 u_{1}(k) \sin \left[u_{1}(k-1) u_{2}(k)\right]+d_{1}(k) \\
y_{2}(k+1)= & -0.1 y_{2}(k)+0.3 y_{2}(k-1)+0.7 u_{1}(k) \\
& +0.4 u_{1}(k-1)+0.8 u_{2}(k)+u_{2}(k-1) \\
& +0.2 y_{2}(k) u_{1}(k) /\left[1+u_{1}^{2}(k)+y_{2}^{2}(k)\right] \\
& +0.1 u_{2}(k) \sin \left(u_{2}^{2}(k-1)\right) \\
& -0.1 \sin \left[0.5 y_{2}^{2}(k-1)\right]+d_{2}(k)
\end{aligned}
$$
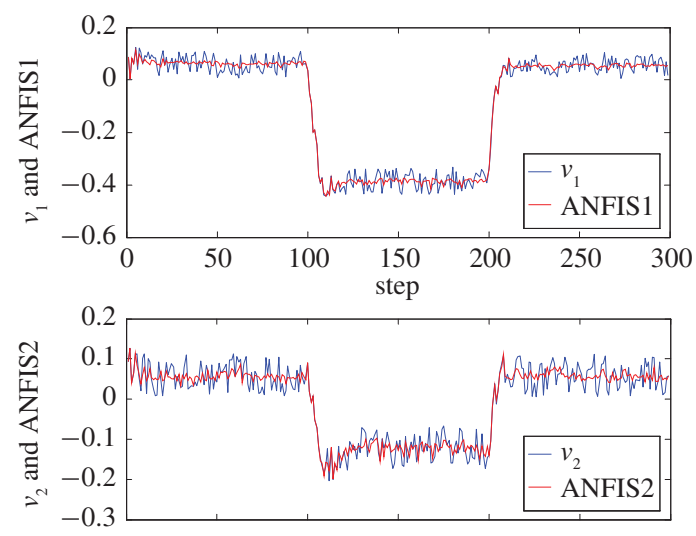

Fig. 15. Virtual unmodeled dynamics and its estimation.
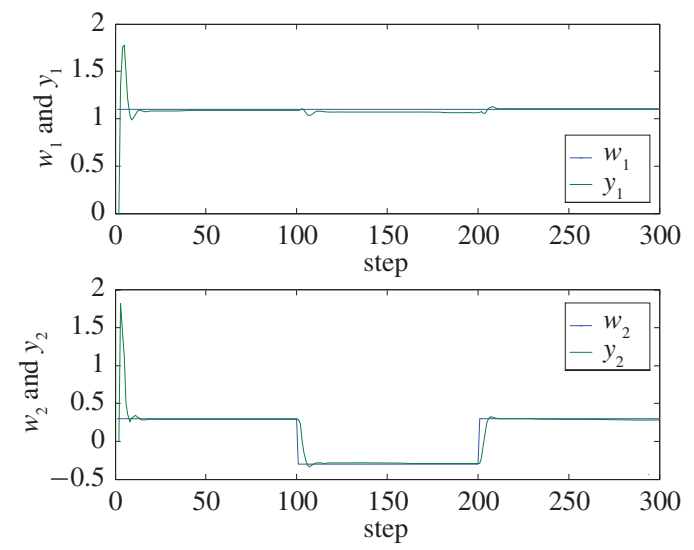

Fig. 16. Responses of system output $\boldsymbol{y}(k)$ and the desired output $\boldsymbol{w}(k)$ when the switching controller is used.

where $d_{1}(k)=1$ and $d_{2}(k)=1.5$ are unknown yet bounded constant disturbances.

It can be seen that the actual system orders are $n_{s}=2$ and $m_{s}=1$. However, we deliberately select the structure orders of the controller-driven model as $n_{g}=n_{a}-1=0$ and $n_{b}=1$. This means that the structure order of the model for the controller design is set to $n_{a}=1$, which is lower than that the actual value. In simulations, other parameters are selected to be the same as those in the first two simulation examples, and the results are shown in Figs. 16-19.

Even though the order of the controller design model is chosen to be lower than that of the system and the system is also subjected to unknown external disturbances, from Figs. 16 and 19 it can be seen that the closed-loop system exhibits large initial tracking error since the linear adaptive controller does not compensate the virtual unmodeled dynamics at the beginning. However, at $k=6$, the nonlinear controller is switched on which compensates the virtual unmodeled dynamics, leading to a better tracking performance for the output of the closed-loop system.

In addition, despite the low order controller design model and unknown external disturbances, from Fig. 19 it can be seen that the virtual unmodeled dynamics of the system can be reasonably well estimated using the proposed estimation algorithm. 

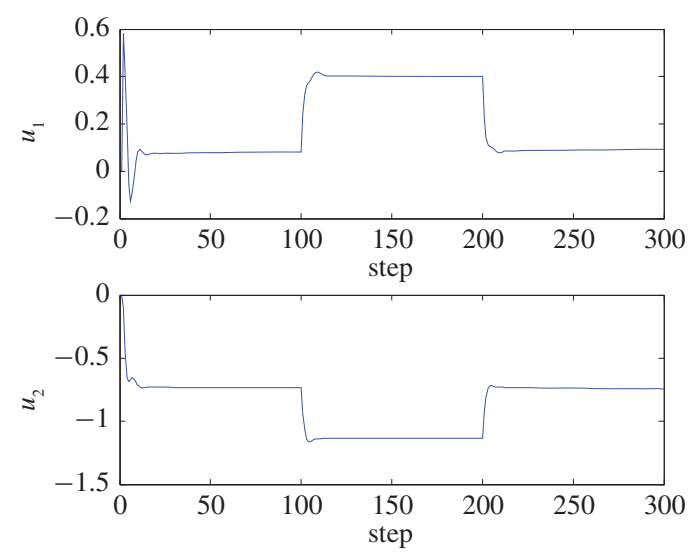

Fig. 17. Control input responses $u_{1}(k)$ and $u_{2}(k)$ when the switching control is used.

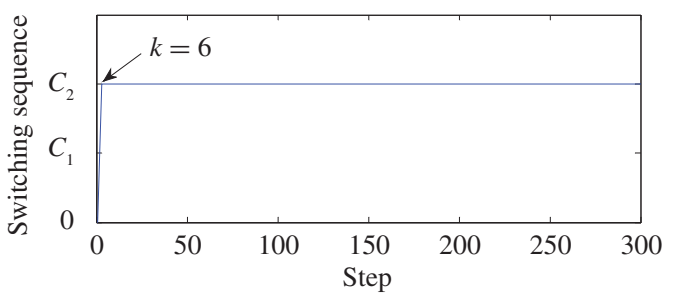

Fig. 18. Switching sequence.
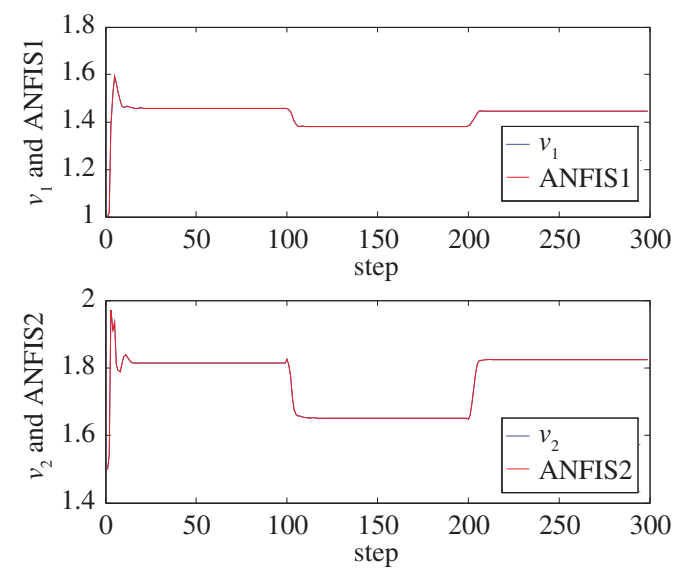

Fig. 19. Virtual unmodeled dynamics $v(k)$ and its estimation.

\section{B. Experimental Results with a Twin-Tank Level Control System}

In this section, we will describe the application results of the proposed method to a twin-level tank control system with two inputs and two outputs as shown in Fig. 20. The actual system consists of nos. 1 and 2 tanks, actuators, a pump, and a valve together with flow-rate and level sensors. The control algorithm is realized using embedded controller as shown in Fig. 21.

In the experiment, the discharging valves 1 and 2 and the inlet valve for the no. 2 tank are kept open. The control inputs for this twin-tank level control system are the valve opening $u_{1}(k)$ and the pulse width modulating ratio $u_{2}(k)$ of the voltage applied to the pump. The outputs are the levels of the no. 1 tank and no. 2 tank denoted by $y_{1}(k)$ and $y_{2}(k)$, respectively.

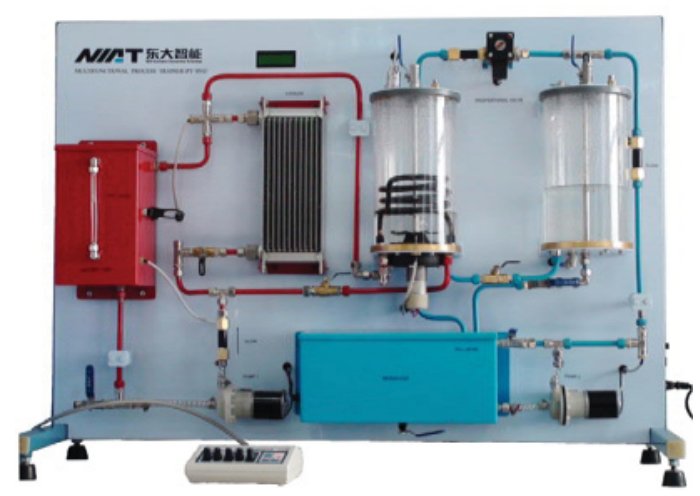

Fig. 20. Twin-level tank.

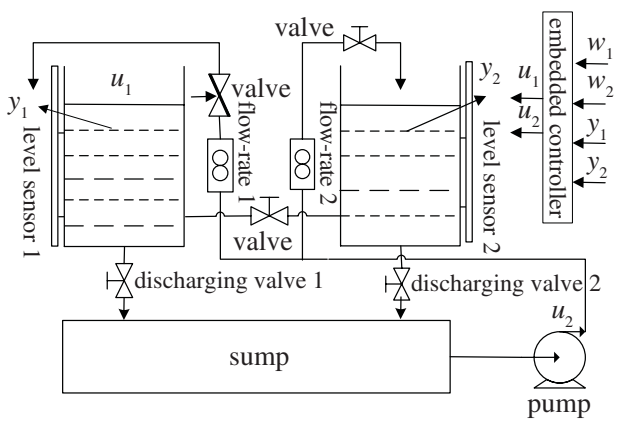

Fig. 21. Structure of the twin-tank level process control system.

Control signal $u_{2}(k)$ is applied to realize the pumping of the water from the sump first to the no. 1 tank then further flow to the no. 2 tank so as to control $y_{2}(k)$. Control input $u_{1}(k)$ is applied to the valve that adjusts the input water flow to the no. 1 tank so as to control its level $y_{1}(k)$. Apparently both level control systems are subjected to strong nonlinearities which cannot be easily modeled mathematically.

To apply the proposed method, first the structure orders of the controller design model are selected to be $n_{a}=2, n_{b}=1$ and the model is taken as linear. This leads to the self-tuning parameter matrix for the linear controller-driven model as follows:

$$
\begin{gathered}
\boldsymbol{G}\left(z^{-1}\right)=\left[\begin{array}{ll}
g_{11}^{0}+g_{11}^{1} z^{-1} & g_{12}^{0}+g_{12}^{1} z^{-1} \\
g_{21}^{0}+g_{21}^{1} z^{-1} & g_{22}^{0}+g_{22}^{1} z^{-1}
\end{array}\right] \\
\boldsymbol{H}\left(z^{-1}\right)=\left[\begin{array}{ll}
h_{11}^{0}+h_{11}^{1} z^{-1} & h_{12}^{0}+h_{12}^{1} z^{-1} \\
h_{21}^{0}+h_{21}^{1} z^{-1} & h_{22}^{0}+h_{22}^{1} z^{-1}
\end{array}\right]
\end{gathered}
$$

where the data vector is defined as

$$
\begin{aligned}
\boldsymbol{x}(k)= & {\left[y_{1}(k), y_{2}(k), y_{1}(k-1), y_{2}(k-1), u_{1}(k), u_{2}(k),\right.} \\
& \left.u_{1}(k-1), u_{2}(k-1)\right]^{\mathrm{T}} .
\end{aligned}
$$

In real-time control, the projection algorithm is used for the estimation of the parameters of the controller-driven model as in (49)-(56). The normalization parameters $\varepsilon_{1}$ and $\varepsilon_{2}$ for the virtual unmodeled dynamics $\overline{\boldsymbol{v}}(k)$ are selected as 0.001 and 0.1 , respectively. For the switching function, we have $M$ given by

$$
\begin{aligned}
2 \gamma(k-1) & =0.002\|x(k-1)\|+0.2, \\
c & =1 .
\end{aligned}
$$




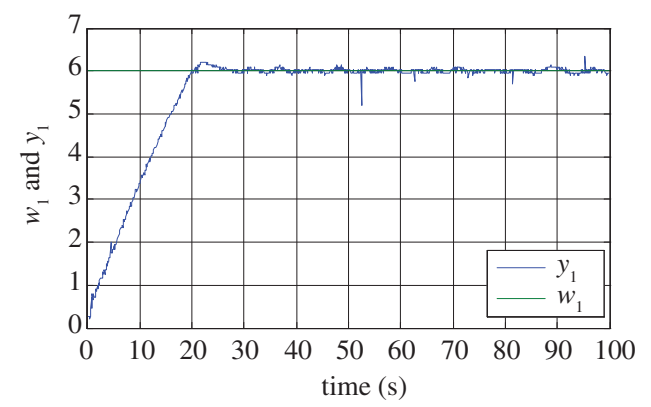

Fig. 22. Response of the no. 1 tank level using the proposed method $\left(w_{1}\right.$ and $\left.y_{1}\right)$.

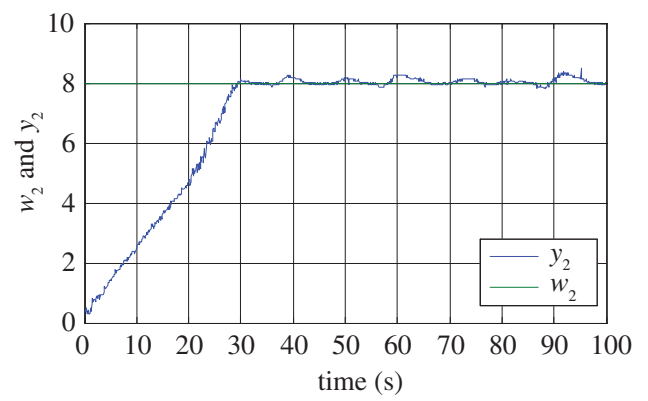

Fig. 23. Response of the no. 2 tank level using the proposed control method $\left(w_{2}\right.$ and $\left.y_{2}\right)$.

The weighting matrices $\boldsymbol{Q}\left(z^{-1}\right)$ and $\boldsymbol{K}\left(z^{-1}\right)$ are given as follows:

$$
\begin{aligned}
\boldsymbol{Q}\left(z^{-1}\right) & =\left[\begin{array}{cc}
0.001 & 0.2 \\
0.1 & 0.001
\end{array}\right]\left(1-z^{-1}\right) \\
\boldsymbol{K}\left(z^{-1}\right) & =\left[\begin{array}{cc}
0.01 & 0 \\
0 & 0.01
\end{array}\right]\left(1-z^{-1}\right) .
\end{aligned}
$$

The desired outputs for this twin-tank level control system are selected as $w_{1}=6 \mathrm{~cm}$ and $w_{2}=8 \mathrm{~cm}$, respectively, with the sampling period being 0.1 second.

The adaptive switching control algorithm in this paper was first programmed using advanced embedded control system toolbox. The codes are then downloaded to the real-time controller so as to execute the required adaptive control. The experimental results are shown in Figs. 22-26.

In Figs. 22 and 23, the actual output responses of the two tank levels are displayed when the proposed adaptive control is applied to the system. Figs. 24 and 25 show the responses of the control inputs, while Fig. 26 gives the switching sequence of the controller. From these figures, it can be seen that a significant improvement has been achieved for the response speed and tracking when the proposed nonlinear adaptive control is applied.

From Figs. 24 and 25 it can be seen that since the nonlinear adaptive control offers dynamical compensation for the virtual unmodeled dynamics, the system has smaller control input magnitudes yet smoother responses as compared to those when the linear adaptive controller is applied. It also shows that less energy would be required for the proposed control method, thereby causing less wearing and reducing the chance of component damage to the actuators.

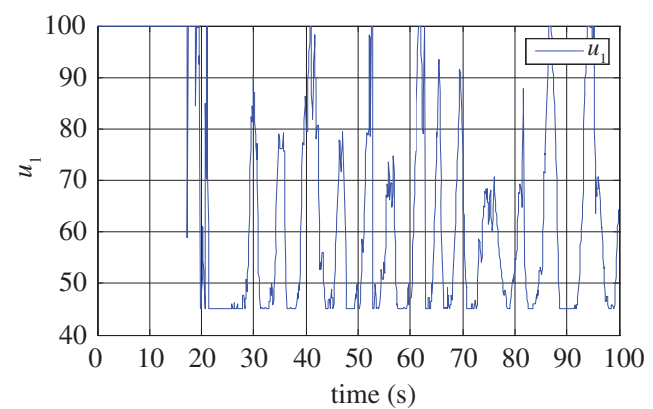

Fig. 24. Response of the input to the no. 1 tank using the proposed control method $\left(u_{1}\right)$.

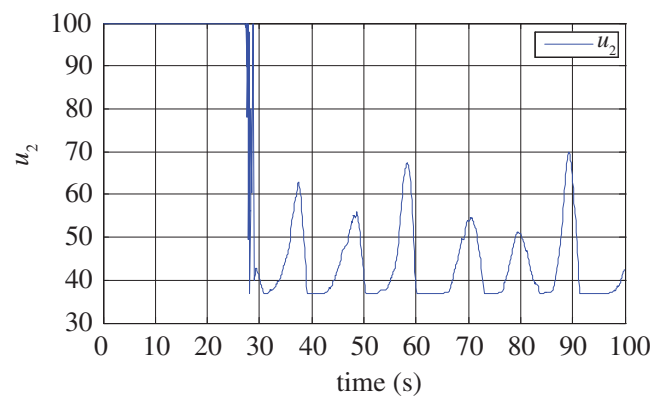

Fig. 25. Response of the input to the no. 2 tank using proposed control method $\left(u_{2}\right)$.

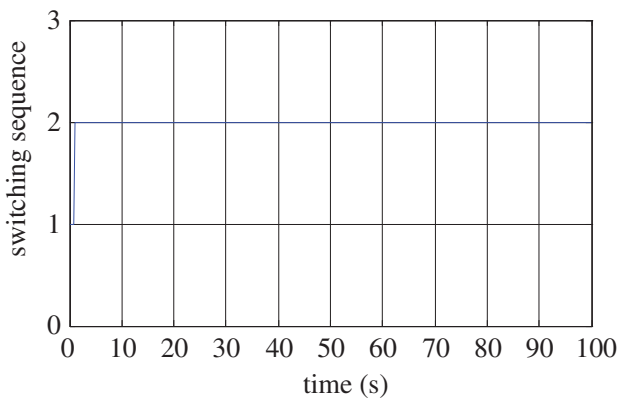

Fig. 26. Switching sequence of the controller.

Fig. 26 displays the switching sequence of the controller, where it can be seen that initially the linear adaptive controller is effective so as to stabilize the closed-loop system. When the system is stabilized, for the most period of time the nonlinear adaptive controller is in action so as to improve the closedloop performance.

\section{CONCLUSION}

In this paper, with the introduction of novel concepts on controller-driven model and virtual unmodeled dynamics, a control design framework was proposed which consists of two controllers, one based on a linear controller-driven model and the other based on a nonlinear controller-driven model. Both models were updated with a proper estimation schemes. The primary objective of the first design based on linear controller-driven model is to stabilize the system, while the main focus of the second one based on nonlinear controllerdriven model is to improve performance. A compensator for the virtual unmodeled dynamics was incorporated with the 
nonlinear controller-driven model with self-tuning parameters. Using a switching mechanism, the two adaptive controllers were integrated to leverage their complementary features. Analysis on stability and convergence has been performed and presented. The proposed adaptive control method has been successfully applied to a twin-tank level control system, in addition to three test examples. Indeed, it can be seen that the proposed adaptive switching control can be applied to a class of multivariable nonlinear systems with unknown model structure and parameters.

\section{ACKNOWLEDGMENT}

The authors would like to thank the editors and the anonymous reviewers for their constructive comments and suggestions on this paper which have helped us to a greatly improved its quality. The authors are grateful to D. Wang for his comments on the technical aspect and some useful discussions of this paper.

\section{REFERENCES}

[1] P. E. McDermott and D. A. Mellichamp, "A decoupling pole-placement self-tuning controller for a class of multivariable process," Opt. Cont. Appl. Methods, vol. 7, no. 1, pp. 55-79, Jan-Mar. 1986.

[2] S. J. Lang, X. Y. Gu, and T. Y. Chai, "A multivariable generalized selftuning controller with decoupling design," IEEE Trans. Automat. Cont., vol. 31, no. 5, pp. 474-477, May 1986.

[3] B. Wittenmark, R. Middleton, and G. Goodwin, "Adaptive decoupling of multivariable systems," Int. J. Cont., vol. 46, no. 6, pp. 1993-2009, 1987.

[4] T. Y. Chai, "Direct adaptive decoupling control for general stochastic multivariable systems," Int. J. Cont., vol. 51, no. 4, pp. 885-909, 1990.

[5] K. Y. Zhu, X. F. Qin, and T. Y. Chai, "A new decoupling design of self-tuning multivariable generalized predictive control," Int. J. Adapt. Cont. Sig. Process., vol. 13, no. 3, pp. 183-196, May 1999.

[6] L. J. Chen and K. S. Narendra, "Nonlinear adaptive control using neural networks and multiple models," Automatica, vol. 37, no. 8, pp. 12451255,2001

[7] Y. Fu and T. Y. Chai, "Nonlinear multivariable adaptive control using multiple models and neural networks," Automatica, vol. 43, no. 6, pp. 1101-1110, Jan. 2007.

[8] Y. Fu and T. Chai, "Neural-network based nonlinear adaptive dynamical decoupling control," IEEE Trans. Neural Netw., vol. 18, no. 3, pp. 921925, May 2007.

[9] T. Y. Chai, L. F. Zhai, and H. Yue, "Multiple models and neural networks based decoupling control of ball mill coal-pulverizing systems," $J$. Process Cont., vol. 21, no. 3, pp. 351-366, Mar. 2011.

[10] M. R. Sanner and E. S. Jean-Jacques, "Gaussian networks for direct adaptive control," IEEE Trans. Neural Netw., vol. 3, no. 6, pp. 837-863, Nov. 1992.

[11] T. Agnoloni and E. Mosca, "Controller falsification based on multiple models," Int. J. Adapt. Cont. Sig. Process., vol. 17, pp. 163-177, Mar. 2003.

[12] M. G. Safonov and T. C. Tsao, "The unfalsified control concept and learning," IEEE Trans. Automat. Cont., vol. 42, no. 6, pp. 843-847, Jun. 1997.

[13] J. C. Spall and J. A. Cristion, "Model-free control of nonlinear stochastic systems with discrete-time measurements," IEEE Trans. Automat. Cont., vol. 43, no. 9, pp. 1198-1210, Sep. 1998.

[14] H. Hjalmarsson, M. Gevers, S. Gunnarsson, and O. Lequin, "Iterative feedback tuning: Theory and application," IEEE Cont. Syst. Mag., vol. 18, no. 4, pp. 26-41, Aug. 1998.

[15] M. C. Campi, A. Lecchini, and S. M. Savaresi, "Virtual reference feedback tuning: A direct method for the design of feedback controllers," Automatica, vol. 38, no. 8, pp. 1337-1346, Aug. 2002.

[16] I. Markovsky and P. Rapisarda, "Data-driven simulation and control," Int. J. control, vol. 81, no. 12, pp. 1946-1959, 2008.

[17] J. Helvoort, B. D. Jager, and M. Steinbuch, "Data-driven multivariable controller design using ellipsoidal unfalsified control," IEEE Syst. Cont. lett., vol. 57, no. 9, pp. 759-762, Sep. 2008
[18] L. Miskovic, A. Karimi, D. Bonvin, and M. Gevers, "Correlation-based tuning of decoupling multivariable controllers," IEEE Trans. Automat., vol. 43, no. 9, pp. 1481-1494, Sep. 2007.

[19] J. S. R. Jang, "ANFIS: Adaptive-network-based fuzzy inference system," IEEE Trans. Syst. Man Cybern., vol. 23, no. 3, pp. 665-685, MayJun. 1993.

[20] H. Wang, A. P. Brown, and C. J. Harris, "One-to-one mapping and its application to neural networks based control systems design," Int. J. Syst. Sci., vol. 27, no. 2, pp. 161-170, 1996.

[21] M. Sugeno and G. T. Kang, "Structure identification of fuzzy model," Fuzzy Sets Syst., vol. 28, no. 1, pp. 15-33, Oct. 1988.

[22] H. G. Zhang and Y. B. Quan, "Modeling identification and control of a class of nonlinear system," IEEE Trans. Fuzzy Syst., vol. 9, no. 2, pp. 349-354, Apr. 2001.

[23] L. X. Wang, "Fuzzy systems are universal approximators," in Proc. IEEE Int. Conf. Fuzzy Syst., San Diego, CA, Mar. 1992, pp. 1163-1170.

[24] G. C. Goodwin and K. S. Sin, Adaptive Filtering Prediction and Control. Englewood Cliffs, NJ: Prentice-Hall, 1984

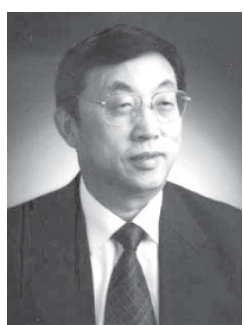

Tianyou Chai (M'90-SM'97-F'08) received the Ph.D. degree from Northeastern University, Shenyang, China, in 1985.

$\mathrm{He}$ is currently a Chair Professor with Northeastern University. He is also the Director of the State Key Laboratory of Synthetical Automation for Process Industries, the National Engineering and Technology Research Center of Metallurgical Automation, and the Department of Information and Science of National Natural Science Foundation of China. He has published two monographs, 84 peer-reviewed papers in international journals, and around 219 papers in international conference proceedings. His current research interests include adaptive controls, intelligent decoupling controls, integrated plant controls and systems, and the development of control technologies with application to various industrial processes.

Prof. Chai is a member of the Chinese Academy of Engineering and a fellow of the International Federation of Automatic Control (IFAC). $\mathrm{He}$ has been invited to give more than 20 plenary speeches at the international conferences of IFAC and IEEE. He has won three prestigious awards of the National Science and Technology Progress, as well as the Technological Science Progress Award from the Ho Leung Ho Lee Foundation in 2002.

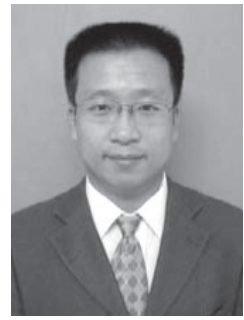

Yajun Zhang was born in Baoji, China, in 1979. He received the M.S. degree in applied mathematics from the Liaoning University of Technology, Jinzhou, China, and the Ph.D. degree in control theory and control engineering from Northeastern University, Shenyang, China, in 2007 and 2011, respectively.

His current research interests include nonlinear fuzzy adaptive control theory, generalized predictive controls, multiple models and switching systems, intelligent decoupling controls, and data-based driven controls and their applications. 


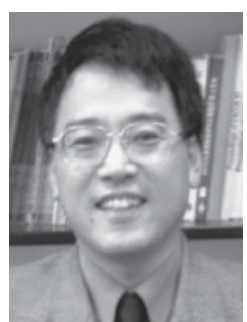

Hong Wang (M'95-SM'05) was born in Beijing, China, in 1960. He received the B.S. degree from the Huainan University of Mining Engineering, Huainan, China, in 1982, and the M.S. and Ph.D. degrees from the Huazhong University of Science and Technology, Huazhong, China, in 1984 and 1987, respectively.

He was a Research Fellow with the University of Salford, Salford; Brunel University, Uxbridge; and Southampton University, Southampton, all in the U.K., from 1988 to September 1992. He then joined the University of Manchester Institute of Science and Technology, Manchester, U.K., in 1992, and has been a Professor in process control with the Control Systems Center since 2002. He also holds a research position with Northeastern University, Shenyang, China, the Huazhong University of Science and Technology, and the Institute of Automation, Chinese Academy of Sciences, Beijing. He has published 200 papers and three books. His current research interests include stochastic distribution controls, fault detection and diagnosis, nonlinear controls, and data-based modeling for complex systems.

Prof. Wang was an Associate Editor of the IEEE TRANSACTIONS ON Automatic Control and currently serves as an Editorial Board Member for the Journal of Measurement and Control, the Transactions of the Institute of Measurement and Control, Impeached Journal of Systems and Control Engineering, Automatica Sinica, and the Journal of Control Theory and Applications.

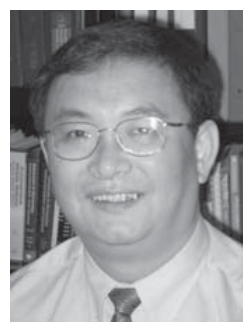

Chun-Yi Su received the Ph.D. degree from the South China University of Technology, Guangzhou, China, in 1990.

$\mathrm{He}$ is currently a Professor and holds the Concordia Research Chair at Concordia University, Montreal, QC, Canada. His current research interests include the application of automatic control theory to mechanical systems, particularly control of systems involving hysteresis nonlinearities.

Prof. Su has served on the editorial boards of several journals, including the IEEE TRANSACTIONS ON AUtOMATIC CONTROL and the IEEE TRANSACTIONS ON CONTROL Systems TeChNOLOGY. He has also served as an organizing committee member for many conferences.

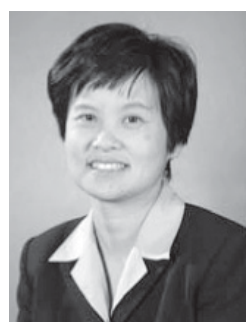

Jing Sun (F'04) received the Ph.D. degree from the University of Southern California, Los Angeles, in 1989, and the B.S. and M.S. degrees from the University of Science and Technology of China, Hefei, China, in 1982 and 1984, respectively.

She was an Assistant Professor with the Department of Electrical and Computer Engineering, Wayne State University, Detroit, MI. She joined Ford Research Laboratory, Dearborn, MI, in 1993 where she was with the Powertrain Control Systems Department. After spending almost ten years in industry, she came back to academia and joined the faculty of the College of Engineering, University of Michigan, Ann Arbor, in 2003, where she is currently a Professor with the Departments of Naval Architecture and Marine Engineering and Electrical Engineering and Computer Science. She holds over 30 U.S. patents and has co-authored a textbook, Robust Adaptive Control. Her current research interests include system and control theory and its application to marine and automotive propulsion systems.

Prof. Sun is one of the three recipients of the IEEE Control System Technology Award in 2003. 